\title{
TAKING A CRITICAL LOOK AT HOLOGRAPHIC CRITICAL MATTER
}

\author{
D.V. Khveshchenko \\ Department of Physics and Astronomy, University of North Carolina, Chapel Hill, NC 27599, U.S.A. \\ E-mail:khvesh@physics.unc.edu
}

Received 4 June 2015; accepted 29 September 2015

\begin{abstract}
Despite a recent flurry of applications of the broadly defined ('non-AdS/non-CFT') holographic correspondence to a variety of condensed matter problems, the status of this intriguing, yet highly speculative, approach remains largely undetermined. Here we expose a number of potential inconsistencies between the previously made holographic predictions and advocate for a compelling need to systematically contrast the latter against the results of alternate, more conventional, approaches as well as experimental data. It is also proposed to expand the list of computed observables and utilize the general relations between them as a further means of bringing the formal holographic approach into a closer contact with the physical realm.
\end{abstract}

Keywords: condensed matter applications of the holographic conjecture

PACS: $71.10 .-\mathrm{W}$

\section{Introduction}

Quantum many-body theory has long been seeking to expand its toolbox of computational techniques, thus allowing one to describe and classify a broad variety of non-Fermi liquid (NFL) states of strongly correlated fermions.

In generic $1 d$ fermion systems, the conventional Fermi liquid behaviour gets (marginally) destroyed by an arbitrarily weak short-ranged repulsive interaction, thereby giving way to the so-called Luttinger behaviour. As one of the hallmarks of the Luttinger regime, the electron propagator exhibits an algebraic decay with distance/time, $G(x) \propto 1 / x^{\Delta}$, governed by an anomalous dimension $\Delta>1$. Moreover, long-range interactions, such as Coulomb, modify the Fermi liquid behaviour even more drastically, resulting in, e. g. the $1 d$ Wigner crystal state where the fermion propagator decays faster than any power law, $G(x) \sim \exp \left(-\# \ln ^{3 / 2} x\right)$.

In higher dimensions, the Fermi liquid is generally believed to be more robust, although it is not expected to remain absolutely stable. While in the case of shortranged repulsive interactions any departures from the Fermi liquid are likely to be limited to the strongcoupling regime, long-ranged couplings can possibly result in the NFL types of behaviour without any threshold.
Of a particular interest are the spectroscopic and transport properties of such emergent critical behaviours as incipient s-, p-, and d-wave charge/spin density waves and orbital current-type instabilities in itinerant (anti)ferromagnets, quantum spin liquids, compressible ('composite fermion') Quantum Hall states, etc. Recently, the focus has also been on the $d>1$-dimensional zero density ('neutral') Dirac/Weyl systems characterized by the presence of isolated points ('nodes') or lines ('arcs') of vanishing quasiparticle energy.

The intrinsic complexity of these systems has long been recognized, prompting the use of such sophisticated techniques as the renormalization group, $1 / \mathrm{N}$ - and $\epsilon$-expansions, Keldysh functional integral and quantum kinetic equation, supersymmetric diffusive and ballistic $\sigma$-models, multi-dimensional bosonization, etc. In spite of all the efforts, however, the overall progress towards a systematic classification of various 'strange' metallic (compressible) states that are often indiscriminately referred to as 'higher dimensional Luttinger liquids' has been rather slow.

In that regard, the recent idea of a (broadly defined) holographic correspondence [1-4] could provide a sought-after powerful alternative technique. Specifically, its widely used 'bottom-up' version may offer an advanced phenomenological framework for 
discovering new and classifying the already known types of NFL behaviour.

Although in much of the pertinent literature the validity of the generalized ('non-AdS/non-CFT') holographic conjecture appears to be taken for granted, it might be worth keeping in mind that the actual status of the entire holographic approach remains anything but firmly established.

Indeed, in most of its applications this bold adaptation of the original 'bona fide' string-holographic correspondence does not seem to be subject to much (or, for that matter, any) of the former's stringent symmetry conditions, as the pertinent non-relativistic systems at finite density and temperature, in general, tend to be neither very strongly coupled nor, conformally, Lorentz or even translationally/rotationally invariant and lack any supersymmetry or even an ordinary gauge symmetry with some rank- $N>1$ (let alone, $N \gg 1$ ) non-abelian group.

While some agreement between the holographic predictions and certain selectively chosen experimental data (usually pertaining to those situations where the extreme strongly-correlated hydrodynamic regime can indeed be attained) has been claimed, there is still no consensus on neither the ultimate implications of such circumstantial evidence nor the general applicability conditions of the holographic approach itself.

The precious few examples of a quantitative agreement between the holographic approach and other (e. g., Monte Carlo) techniques involve some carefully tailored gravity duals (whose physical nature still remains rather obscure). Conspicuously, though, the best quantitative agreement has been achieved in those cases where the allegedly all-important $N \gg 1$ condition does not seem to play much of a role, as in the $2 d$ Bose-Hubbard (or quantum $X Y$-) model with $N=2$ [5-9].

In other cases, under a closer inspection the purported agreement appears to be largely limited to a purely visual similarity between the results of some (for the most part, numerical) calculations and certain selected sets of the available experimental data.

For one, in Ref. [10-13] the holographically computed optical conductivity was claimed to agree (over less than half of a decade, $2<\omega \tau<8$ ) with the enigmatic power-law decay, $\sigma(\omega) \sim \omega^{-2 / 3}$, observed in the normal state of the superconducting cuprates (BSCYCO), pnictides, and certain heavy fermion materials, often up to the energies of $\mathrm{eV}$ order. Notably, the original claim was not corroborated by the later analysis of Ref. [14] and was also argued to be intermittent with the 'more universal' $\sim 1 / \omega$ behaviour [15, 16 .

Also, while being traditionally wordy and profuse on the auxiliary technical details, many of the works on holography end up with rather simple scaling relations as their final answers, thereby suggesting that there might be more economic and physically illuminating ways of obtaining such results.

Thus, in order for its status to be definitively ascertained, the holographic approach needs to be assessed critically and applied to those systems where a preliminary insight can be (or has already been) gained by some alternative means, so that a systematic comparison with the holographic results can be made. Also, in order to demonstrate their predictive power the holographic calculations would have to be performed for as many observables as possible and then applied to the host of experimental data on the documented NFL materials. This communication takes a step towards filling in the gap.

\section{Practical holography of condensed matter systems}

In its original formulation, the holographic principle postulates that certain $d+1$-dimensional 'boundary' field theories allow for a dual description involving, alongside other 'bulk' fields, $d+2$-dimensional gravity. Moreover, when the boundary theory is strongly coupled, the higher-dimensional gravity appears to allow for a semiclassical treatment, thus facilitating a powerful new approach to the problem of strong interactions.

So far, the holographic approach has been opportunistically applied to a variety of systems which includes 'strange' Fermi and Bose metals describing quantum-critical $U(1)$ and $Z_{2}$ spin liquids, itinerant (anti)ferromagnets, quantum nematics, Mott transitions in lattice and cold atom systems, Hall effect, graphene, etc.

On the gravity side, the system in question would be characterized by a (weakly) fluctuating background metric $g_{\mu v}=g_{\mu v}^{(0)}(r)+\delta g_{\mu v}(t, \vec{x}, r)$ determining the interval

$$
\mathrm{d} s^{2}=g_{t t} \mathrm{~d} t^{2}+g_{r r} \mathrm{~d} r^{2}+\sum_{i j} g_{i j} \mathrm{~d} x^{i} \mathrm{~d} x^{j} .
$$

The early applications of the holographic approach revolved around a handful of the classic 'black brane' solutions, such as the Reissner-Nordstrom AdS (antide-Sitter) black hole with the metric

$$
g_{t t} \sim-\frac{f(r)}{r^{2}}, g_{r r} \sim \frac{1}{r^{2} f(r)}, g_{i j}(r) \sim \delta_{i j} \frac{1}{r^{2}},
$$

where the emblackening factor $f(r)$ vanishes at the horizon of radius $r_{\mathrm{h}}$ which is inversely proportional (ostensibly, similar to the case of the Schwarzschild black 
hole in the asymptotically Minkowski space-time, despite the variable's $r$ being the inverse of the actual radius in the $d+1$-dimensional bulk space) to the Hawking temperature $T$ shared by the bulk and boundary degrees of freedom. The explicit form of this function depends on how the gravito-electromagnetic background is described.

In the black brane geometry of the minimal Einstein-Maxwell theory, one has $f_{\mathrm{EM}}(r)=1-\left(r / r_{\mathrm{h}}\right)^{d+1}$, whereas in the Dirac-Born-Infeld (DBI) theory with the Lagrangian

$$
L_{\mathrm{DBI}}=\sqrt{-\operatorname{det}\left[g_{\mu \nu}+F_{\mu \nu}\right]}
$$

geared to the strong-field limit $f_{\mathrm{DBI}}(r)=\sqrt{1+(e n)^{2} r^{2 d}}$, en being the boundary density of electric charge.

In the early works on the subject, the metric (2) was claimed to provide a potential gravity dual to the class of strongly correlated condensed matter problems - most notably, heavy fermion materials and cuprates - which are believed to manifest a certain 'semi-locally critical' behaviour [17-23]. However, soon thereafter it was realized that the corresponding physical scenario appears to be too much limited to encompass more general types of the reallife NFLs, so the focus shifted towards a broader class of geometries.

Further attempts of 'reverse engineering' have brought out such Lorentz non-invariant metrics as the Shroedinger, Lifshitz, helical Bianchi, etc. Amongst them, a particular attention has been paid to the static, diagonal, and isotropic metrics with algebraic radial dependence

$$
g_{t t} \sim-\frac{1}{r^{2 \alpha}}, g_{r r} \sim \frac{1}{r^{2 \beta}}, g_{i j} \sim \frac{1}{r^{2 \gamma}} .
$$

These exponents are defined modulo a change of the radial variable $r \rightarrow \rho^{\delta}$ resulting in the substitution

$$
\alpha \rightarrow \alpha \delta, \beta \rightarrow \beta \delta-\delta+1, \gamma \rightarrow \gamma \delta .
$$

Unless $\gamma=0$, the metric (4) is conformally equivalent to the one

$$
g_{t t} \sim r^{2(\theta / d-z)}, g_{r r}=g_{i i} \sim r^{2(\theta / d-1)}
$$

characterized by only two parameters

$$
\theta=d \frac{1-\beta}{1-\beta+\gamma}, \quad z=\frac{1+\alpha-\beta}{1-\beta+\gamma}
$$

which describe a family of 'hyperscaling-violating' (HV) backgrounds [24-33] where the dynamical exponent $z$ controls the boundary excitation spectrum $\omega \propto q^{z}$, while $\theta$ quantifies a non-trivial scaling of the interval $\mathrm{d} s \rightarrow \lambda^{\theta / d} \mathrm{~d} s$, the scaling-(albeit not Lorentz-) invariant ('Lifshitz') case corresponding to $\theta=0$.

The finite- $T$ version of the HV metric can be constructed by decorating (6) with the additional factor $f_{\mathrm{HV}}(r)=1-\left(r / r_{\mathrm{h}}\right)^{d+z-\theta}$, akin to Eq. (2), which introduces the black brane's horizon located at $r_{\mathrm{h}} \sim T^{-1 / z}$.

The physically sensible values of $z$ and $\theta$ are expected to satisfy the all-important 'null energy conditions'

$$
(d-\theta)[d(z-1)-\theta] \geq 0,(z-1)(d+z-\theta) \geq 0
$$

signifying a thermodynamic stability of the corresponding geometry.

The HV metrics have been extensively discussed in the content of various generalized gravity theories, including those with massive vector fields as well as the Einstein-Maxwell dilaton (EMD) theory which includes an additional scalar field, alongside the cosmological constant term [24-33]

$$
L_{\mathrm{EMD}}=\frac{1}{2 \kappa^{2}}\left[R+\frac{d(d+1)}{L^{2}}\right]-\frac{(\partial \phi)^{2}}{2}-U(\phi)-\frac{V(\phi)}{2 e^{2}} F_{\mu v}^{2} .
$$

In its minimal version, both the dilaton potential $U(\phi)$ and the effective gauge coupling $V(\phi)$ are given by some exponential functions of $\phi$.

At the (semi)classical level, gravitating matter added to the EMD Lagrangian (9) can be described in terms of its energy-momentum tensor and electric current

$$
T_{\mu \nu}=(E+P) u_{\mu} u_{v}+P g_{\mu v} J_{\mu}=e n u_{\mu}
$$

where en, $E, P, u_{u}$ are the charge and energy densities, pressure, and (covariant) local velocity, respectively. The first law of thermodynamics then relates the above quantities as follows:

$$
E+P=S T+\mu n,
$$

where $S$ is entropy density and $\mu=\left.e A_{t}(r)\right|_{r \rightarrow 0}$ is the chemical potential. In the particle-hole symmetric ('neutral') system $\mu=0$ and the equation of state reads $E=(d-\theta) P / z$.

The HV solutions (6) have also been obtained by taking into account a back-reaction of matter on the background geometry [34, 35]. Such analyses would typically use the Fermi distribution when summing over the occupied fermion states, thereby achieving a partial account of the (Hartree-type) effects of the Fermi statistics, while leaving out more subtle (exchange and correlation) ones.

However, for an already chosen gravitational background the customary way of introducing a finite 
charge density into the holographic scheme is by embedding a D-brane into such geometry and treating it in the probe approximation (no back-reaction). The pertinent dynamics is then described by the DBI action (3) with the background electric field

$$
F_{r t}=\partial_{r} A_{t}=\sqrt{\frac{\left|g_{t t}\right| g_{r r}(e n)^{2}}{\prod_{i} g_{i i}+(e n)^{2}}} .
$$

The DBI approach has been used to study thermodynamics of the HV theory. In that regard, in Refs. [36-38] the specific heat of a finite density ('charged') system was found to scale with temperature as

$$
C_{\mathrm{DBI}, \text { charged }} \sim T^{-2 \theta / z d} .
$$

Being primarily interested in the limit $z \rightarrow \infty$, $-\theta / z \rightarrow$ const, the authors did not seem to be particularly concerned with the implications of this result, including its apparent inapplicability in the potentially physically relevant case of $\theta=d-1>0$ (see below).

Moreover, even for $\theta=0$ Eq. (12) differs from the expression obtained in the earlier work of Refs. [39, 40] where the standard ('black-body') leading term $\sim T^{d / z}$ was deliberately discarded in favour of the subdominant (yet, charge density dependent) one, $\sim T^{2 d / z} /$ en.

Nevertheless, Eq. (12) can be rationalized by comparing it to the result of a direct calculation for the HV metric (6) and $\mu=0$ :

$$
\begin{aligned}
C_{\mathrm{DBI}, \text { neutral }} \sim \frac{\partial}{\partial T} \int_{0}^{r_{h}} \mathrm{~d} r \sqrt{-\operatorname{detg}_{\mu v}} \\
\sim T^{(d-\theta-2 \theta / d) / z} .
\end{aligned}
$$

Physically, this expression can also be recognized as the number, rather than the charge density $n(T)$ of thermally excited carriers (of either sign).

One subtle point is that in the charged case it is not the latter but the charge density that gets replaced with a finite value at not too high temperatures. Based on that insight, the entire temperature dependence in Eq. (12) should then be attributed to the effective ( $T$ dependent) charge

$$
e \sim T^{2 \theta / d z},
$$

while the charge density itself scales as the ratio between Eqs. (12) and (13):

$$
e n=C_{\mathrm{DBI}, \text { neutral }} / C_{\mathrm{DBI}, \text { charged }} \sim T^{(d-\theta) / z} .
$$

The temperature dependence (13) would also be shared by the concomitant thermal entropy

$$
S_{\mathrm{DBI}} \sim n \sim T^{(d-\theta-2 \theta / d) / z} .
$$

Notably, this result of a straightforward thermodynamic calculation appears to be at odds with both the naive estimate

$$
S_{\mathrm{BH}}=\frac{1}{4} A \sim r_{\mathrm{h}}^{-d} \sim T^{d / z}
$$

for the Bekenstein-Hawking entropy, a black $d$-brane of radius $r_{\mathrm{h}}$, as well as the entanglement entropy

$$
S_{\text {ent }} \sim T^{(d-\theta) / z},
$$

the relation that would often be quoted ad hoc with regard to the empiric interpretation of the parameter $\theta$ as a 'defect of dimension' that yields the effective dimension $d_{\text {eff }}=d-\theta$ in the above expression for $S_{\mathrm{BH}}$.

The proper choice of $\theta$ has been extensively discussed in the context of fermionic entanglement entropy which points to the value $\theta_{\mathrm{f}}=d-1$, consistent with the notion of the Fermi surface as a $d$-1-dimensional manifold spanning the tangential directions in the reciprocal (momentum) space of the boundary theory [41, 42].

In fact, by adhering to the above value one chooses to treat the HV system in question as fermionic and, therefore, must use the fermion quasiparticle dispersion $\omega \sim\left(k-k_{\mathrm{F}}\right)^{z \mathrm{f}}$ to determine the value of the dynamical index. In what follows, the metric with the parameters $z=z_{\mathrm{f}} \theta=d-1$ will be called 'Model I'.

Alternatively, one could treat the system as bosonic and use the value $z_{\mathrm{b}}$ deduced from the dispersion of the bosonic mode, $\omega \sim k^{z}$. A straightforward choice for the HV parameter would then be $\theta=0$, thereby reducing the corresponding metric back to the Lifshitz one (hereafter, 'Model II').

Yet another possibility corresponds to choosing $z=z_{\mathrm{f}}$ and $\theta=d\left(1-z_{\mathrm{f}} / z_{\mathrm{b}}\right)$, which choice will be called 'Model III'. In fact, the two latter metrics are related by a conformal transformation, so some of the results turn out to be the same in both cases. And, lastly, there also exists a choice $z=z_{\mathrm{b}}$ and $\theta=d-z_{\mathrm{b}} / z_{\mathrm{f}}$ which will be referred to as 'Model IV' below.

\section{Scaling properties of hyperscaling-violating systems}

The above exposition of the physically incomprehensible holographically computed specific heat suggests that any substantive comparison with the calculations performed by alternate techniques would have to involve more than one quantity. 
Indeed, a power-law behaviour of the specific heat is just one of the many scaling laws which describe quantum-critical systems. The list of other observables includes (tunneling) density of states, charge, current, and spin susceptibilities, electrical, thermal, and spin conductivities, shear and bulk viscosities, etc.

In the quantum-critical regime of a massless $(m=0)$ and particle-hole symmetric (or neutral, $\mu=0$ ) system, the single most important scale is set by temperature $T$ or frequency $\omega$, whichever is greater.

The anticipated algebraic behaviour of a physical observable $A$ is then fully characterized by its scaling dimension $[A]$, namely:

$$
A(\omega, T) \sim \max |T, \omega|^{\Delta A}, \Delta_{A}=[A] / z .
$$

Although in the following discussion no distinction is made between the exponents controlling the frequency and temperature dependences, this point will be addressed later.

Once a new scale enters the game, the pure algebraic dependences would only hold at high enough frequencies and/or temperatures, while at smaller $\omega$ or $T$ any pure power-law gets complemented by a universal function of the ratios between $T$ and all the competing scales $(m, \mu$, etc.). Also, in the case of the vanishing exponent one can encounter a logarithmic dependence $\sim \log \max |T, \omega|$ stemming from either the quantum Anderson localization or the classical 'long-tail' behaviour [43].

The scaling analysis begins with a proper assignment of the scaling dimensions under transformation of the space-time coordinates in the boundary theory.

The basic scaling dimensions of the space-time coordinates and their conjugate energies/momenta take their natural values

$$
\left[x_{i}\right]=-\left[k_{i}\right]=-1,[t]=-[\epsilon]=-[\mu]=-[T]=-z
$$

consistent with the underlying dispersion relation $\omega \sim k^{z}$, whereas those of the gauge potential differ from the above values by the dimension of the effective charge (14)

$$
\left[A_{i}\right]=\left[k_{i} / e\right]=1-2 \theta / d,\left[A_{0}\right]=[\mu / e]=z-2 \theta / d .
$$

For comparison, in Ref. [49] instead of attributing the 'subleading' term $\phi=2 \theta / d$ in the above expressions (cf. Eq. (3.6) in Ref. [49]) to the dimension of the electric charge (14), thus distinguishing between the number and charge densities (or, for that matter, $\mu$ and $A_{0}=\mu / e$ which Ref. [49] makes no distinction of), the gauge sector was assigned its own HV parameter $\theta_{\mathrm{m}}$.
Moreover, it was argued in Ref. [49] that introducing an extra parameter (either $\theta_{\mathrm{m}}$ or $\phi$ ) in addition to $z$ and $\theta$ is necessary for the proper description of a charged system with the HV geometry.

As yet another generalization, in Ref. [49] the spatial dimension of the gauge and/or matter degrees of freedom $d_{\mathrm{s}}$ was allowed to be different from that of the gravitational ones. However, the dimensions of the energy densities (as well as entropies) in the gravitational and gauge/matter sectors can still be made equal, provided that the following condition is satisfied:

$$
\theta=d-d_{\mathrm{s}}+\theta_{\mathrm{m}}+\phi
$$

In fact, for $d=d_{\mathrm{s}}$ and the original DBI action (3) this condition would be impossible to meet for any $\theta \neq 0$, as the pertinent value of the gauge HV parameter is $\theta_{\mathrm{m}, \mathrm{DBI}}=d_{\mathrm{s}} \theta / d[49]$. The overall entropy then consists of the two different contributions given by Eqs. (16) and (17), the latter being dominant only in the customary holographic 'large N' limit (albeit not at the lowest temperatures for a physically relevant $\theta>0$ ).

This observation exposes an intrinsic deficiency of the DBI approach when it is applied to the standard (i. e., 2-parameter) charged HV systems where no 3rd independent parameter (either $\phi$ or $\theta_{\mathrm{m}}$ ) exists. Its purported applications to the condensed matter systems with $N \sim 1$ may, therefore, require extra caution.

Such subtleties aside, Eqs. (18) and (19) yield the derivative scaling relations

$$
\begin{aligned}
& {\left[v_{i}\right]=\left[x_{i} / t\right]=z-1,\left[\nabla_{i} T\right]=z+1,} \\
& {\left[\mathcal{E}_{i}\right]=\left[A_{i} / t\right]=\left[A_{0} / x_{i}\right]=z+1-2 \theta / d,} \\
& {\left[B_{i}\right]=\left[A_{j} / x_{k}\right]=2-2 \theta / d,}
\end{aligned}
$$

where $v_{i}, A_{i}, \varepsilon_{i}, B_{i}$ are the velocity, vector potential, electric and magnetic fields, respectively.

The dimensions of the energy and number densities can be read off directly from Eq. (13):

$$
[E]=[P]=\left[T_{t t}\right]=\left[T_{i j}\right]=[\mu n]=z+d-\theta-2 \theta / d .
$$

Then, in order for the boundary action to maintain scale invariance, a spatial integration must be thought of as contributing the extra dimension

$$
[\mathbf{d x}]=-[n]=-d+\theta+2 \theta / d,
$$

under this convention the total (quasi)particle number $\int n \mathbf{d x}$ is dimensionless and, therefore, conserved. In contrast, the total charge $\int e n \mathbf{d x}$ appears to scale with the non-vanishing dimension (14) imposed by the 'running' dilaton-dependent gauge coupling. 
Granted that in the holographic literature the particle number and charge densities would often be used interchangeably, a potentially important difference between the two cannot be ignored when thermal, thermo-electrical, or stress-related responses are studied, alongside the purely electrical one.

Also, while in a typical holographic setting one would invariably view the electric charge as a conserved quantity, the above discussion suggests that it should have really been the particle number instead. Indeed, the popular interpretation of the bulk radial variable $r$ (with its natural scaling dimension $[r]=-1$ ) as a renormalization group parameter [1-4] would be much more consistent with the notion of a flowing (hence, dimensionful and, therefore, $r$-dependent) renormalized charge, rather than a scale-dependent total particle number.

Continuing with the assignment of dimensions, those of the electrical current and the remaining components of the stress-energy tensor can be readily deduced from the conservation laws

$$
e \frac{\partial n}{\partial t}+\frac{\partial J_{i}}{\partial x_{i}}=0, \frac{\partial T_{t t}}{\partial t}+\frac{\partial T_{i t}}{\partial x_{i}}=0, \frac{\partial T_{t i}}{\partial t}+\frac{\partial T_{j i}}{\partial x_{j}}=0
$$

In this way, one obtains

$$
\begin{aligned}
& {\left[J_{i}\right]=d+z-1-\theta,} \\
& {\left[T_{t i}\right]=d-\theta+1-2 \theta / d,} \\
& {\left[Q_{i}\right]=\left[T_{i t}\right]=d+2 z-1-\theta-2 \theta / d,}
\end{aligned}
$$

where $Q_{i}$ is the thermal current.

It is also worth pointing out that in the absence of the Lorentzian symmetry the stress-energy tensor becomes non-symmetrical (cf. with Refs. [44-48] which dealt exclusively with the case of $\theta=0$, though).

The density susceptibility $\chi$ (related to the charge one by the factor $e^{2}$ ), electrical $\sigma$ and thermal $\kappa$ conductivities are then characterized by the following dimensions:

$$
\begin{aligned}
& {[\chi]=\left[E / \mu^{2}\right]=d-z-\theta-2 \theta / d,} \\
& {[\sigma]=\left[J_{i} / \mathcal{E}_{i}\right]=d-2-\theta+2 \theta / d,} \\
& {[\kappa]=\left[Q_{i} / \nabla_{i} T\right]=d+z-2-\theta-2 \theta / d .}
\end{aligned}
$$

The thus-found dimensions of the thermodynamic and kinetic coefficients allow one to construct their dimensionless universal ratios which are scale invariant (hence, constant for $\mu \ll T$ ). Amongst those are the standard Wilson and Wiedemann-Franz ratios whose vanishing dimensions follow from the above Eqs. (13), 14 and (24)-(26):

$$
\frac{\chi T}{C}=\text { const }, \quad \frac{e^{2} \kappa}{\sigma T}=\text { const } .
$$

Should, however, a new scale emerge, these ratios, albeit remaining dimensionless, would no longer remain constant. In fact, they may deviate strongly from their Fermi liquid values, thereby signalling, e. g., the formation of a strongly correlated (hydrodynamic) quantum-critical state.

It is worth pointing out that Eq. (28) and alike appear to be remarkably robust with regard to changing the assignments of the basic dimensions given by Eqs. (18) and (19) (in the original version of this note, the same ratios were obtained under two more different assignments).

As a means of lending further support to the above scaling relations, one can also reproduce the dimensions (25) and (26) of the electrical and thermal conductivities from the Kubo formulae

$$
\sigma=\frac{1}{\omega} \operatorname{Im} \int \mathbf{d} \mathbf{x} \int_{0}^{\infty} \mathrm{d} t \mathrm{e}^{\mathrm{i} \omega t}\langle[J(t, x), J(0,0)]\rangle
$$

and

$$
\kappa=\frac{1}{\omega T} \operatorname{Im} \int \mathbf{d} \mathbf{x} \int_{0}^{\infty} \mathrm{d} t \mathrm{e}^{\mathrm{i} \omega t}\langle[Q(t, x), Q(0,0)]\rangle,
$$

where the use of the scaling rule (22) is instrumental. Also, while being irrelevant for the general scaling properties, a practically important distinction has to be made between the formally defined thermal conductivity and that computed under the condition of vanishing electric current, the setup that more faithfully represents the actual measurement. The latter is given by the expression

$$
\bar{\kappa}=\kappa-T \sigma \alpha^{2},
$$

where the thermopower (Seebeck coefficient) can be obtained from the Mott relation, $\alpha \sim T \mathrm{~d} \ln \sigma / \mathrm{d} \mu$.

As yet another independent check, the shear viscosity

$$
\eta=\frac{1}{\omega} \operatorname{Im} \int \mathbf{d} \mathbf{x} \int_{0}^{\infty} \mathrm{d} t \mathrm{e}^{\mathrm{i} \omega t}\left\langle\left[T_{x y}(t, x), T_{x y}(0,0)\right]\right\rangle
$$

features the dimension

$$
[\eta]=d-\theta-2 \theta / d,
$$

which, together with Eq. (16), guarantees that the celebrated viscosity-to-entropy ratio $\eta / S$ is indeed dimensionless. 
It can also be easily seen that the dimensions are consistent with the classical (Einstein's) relations

$$
\sigma=e^{2} \chi D, \eta=D(E+P) / \nu^{2},
$$

where the diffusion coefficient $D=v^{2} / d \Gamma$ contains a scattering rate $\Gamma$ expected to assume the universal linear form

$$
\Gamma \sim T
$$

in the quantum-critical regime, thereby allowing one to link the kinetic and thermodynamic coefficients together [50].

Also, observe that the ratio between Eq. (22)

$$
\frac{\eta e^{2} v^{2}}{\sigma T^{2}}=\mathrm{const}
$$

is dimensionless and, therefore, constant in the neutral massless case. As such, it should be contrasted against the proposal

$$
\frac{\eta}{\sigma T^{2 / 2}}=\text { const }
$$

which was put forward for $\theta=0$ in Ref. [51]. Their obvious discrepancy (even in this limit) stems from the improper account of the velocity's dimension in Ref. [51].

In the charged case $(\mu \gg T)$, by using $\chi_{\mathrm{c}}=\mathrm{d} n / \mathrm{d} \mu$ and Eq. (10) one can cast the conductivity in the form

$$
\sigma \sim \frac{e^{2} v^{2} n}{\mu \Gamma}
$$

Should the rate $\Gamma$ then happen to be linear, as in Eq. (27), the conductivity would exhibit the ubiquitous in strongly correlated systems $\sim 1 / T$ behaviour [52], seemingly in agreement with various scenarios of the cuprates and other 'strange metals' that emphasize their proximity to one or another putative quantumcritical point.

The above scaling relations can also be generalized to include anisotropic spatial geometries. In the simplifying case of a unidirectional rotationally anisotropic metric [53-55]

$$
g_{t t} \sim-r^{2 \theta / d-2 z}, g_{\|,\|, \|} \sim r^{2 \theta / d-2 \omega}, g_{r r}=g_{\perp, \perp} \sim r^{2 \theta / d-2}
$$

the scaling dimensions read

$$
\begin{aligned}
& {[t]=-z,\left[x_{\|}\right]=-1,\left[x_{\perp}\right]=-w,} \\
& {\left[A_{\|}\right]=w-2 \theta / d,\left[A_{\perp}\right]=1-2 \theta / d,} \\
& {\left[\mathcal{E}_{\|}\right]=z+\omega-2 \theta / d,\left[\mathcal{E}_{\perp}\right]=1+z-2 \theta / d,}
\end{aligned}
$$

$$
\begin{aligned}
& {\left[J_{\|}\right]=d+z-1-\theta,\left[J_{\perp}\right]=d+z+w-2-\theta,} \\
& {[n]=d+w-1-\theta-2 \theta / d,[B]=1+w-2 \theta / d,}
\end{aligned}
$$

where $B$ is a magnetic field perpendicular to both $\vec{E}$ and $\vec{J}$.

Choosing the axes $x$ and $y$ along the $\|$ and one of the $d-1 \perp$ directions, respectively, one obtains

$$
\begin{aligned}
& {\left[\sigma_{x x}\right]=d-1-w-\theta+2 \theta / d,} \\
& {\left[\sigma_{x y, y x}\right]=d-2-\theta+2 \theta / d,} \\
& {\left[\sigma_{y y}\right]=d+w-3-\theta+2 \theta / d .}
\end{aligned}
$$

In the charged case, all the components are expected to be proportional to the density, as the Hall response of a particle-hole symmetric system vanishes identically. However, the common density factors cancel out in the Hall angle

$$
\cot \theta_{\mathrm{H}}=\frac{\sigma_{x x}}{\sigma_{x y}} \sim \frac{e n}{B \sigma_{x x}} \sim B^{-1} T^{(2 / z)(1-\theta / d)},
$$

where the linear proportionality of $\sigma_{x y}$ to a weak magnetic field has also been taken into account.

Equations (36)-(39) agree with the results of Ref. [53-55] where only the case of $\theta=0$ was considered. A further generalization to the fully anisotropic case would also be quite straightforward.

It was concluded in Refs. [53-55] that both goals of reproducing the linear resistivity and quadratic Hall angle characteristic of, e. g., the behaviour found in the superconducting cuprates cannot be achieved simultaneously, regardless of the choice of $z$ and $w$.

For instance, by choosing $z=1, w=1 / 2, \theta=0$ one does obtain $\sigma_{x x} \sim 1 / T, \cot \theta_{\mathrm{H}} \sim T^{2}$ in the charged case, although it can only come at the expense of acquiring a strong spatial anisotropy (now $\sigma_{y y} \sim 1 / T^{2}$, independent of $w$ or $d$ ).

One can also check that having yet another available parameter $\theta$ does not change the above conclusions. For instance, in the isotropic charged system $(z=w=1)$ one can get $\sigma_{x x, y y} \sim 1 / T$ by simply choosing $\theta=d / 2$, but then the concomitant Hall angle is $\cot \theta_{\mathrm{H}} \sim T$.

It is also instructive to compare the above scaling dimensions to the predictions of the holographic 'membrane paradigm' which offers simple integral expressions for such important thermodynamic characteristics as charge susceptibility [56]

$$
e^{2} \chi=\left(\int_{0}^{r_{\mathrm{h}}} \mathrm{d} r \sqrt{\frac{g_{r r}\left|g_{t t}\right|}{\Pi_{i} g_{i i}}}\right)^{-1}
$$


or enthalpy density

$$
E+P=\left(\int_{0}^{r_{\mathrm{h}}} \frac{\mathrm{d} r}{g_{x x}} \sqrt{\frac{g_{r r}\left|g_{t t}\right|}{\prod_{i} g_{i i}}}\right)^{-1} .
$$

For the HV geometry (6) Eq. (40) yields

$$
e^{2} \chi \sim T^{(d-\theta-z+2 \theta / d) / z}
$$

which fully agrees with (14) and (24).

In contrast, the result of computing Eq. (41)

$$
E+P \sim T^{(d-\theta-z+2) / z}
$$

is clearly at odds with Eq. (21) even for $\theta=0$, as long as $z \neq 1$.

One can readily check that for any $\theta$ such discrepancy cannot be fixed by adding any powers of the velocity and, if taken at its face value, questions the validity of Eq. (41).

Moreover, the 'membrane paradigm' approach offers a closed expression for the WF ratio [51]

$$
\frac{e^{2} \kappa}{T \sigma}=\left(\frac{E+P}{T n}\right)^{2}
$$

Physically, the WF ratio can be viewed as a measure of the energy dependence of the dominant scattering rate. The classic WF law stating a constancy of this ratio would be expected to hold in any regime dominated by (quasi)elastic scattering, including, e. g., the case of electron-phonon scattering either well below or well above the Debye temperature.

In the important case of a zero-density 'relativistic' system with $z=1$, the energy current appears to be identical to the momentum. As a result, the thermal conductivity becomes infinite in the absence of momentum relaxation, thus making the $\mathrm{WF}$ ratio diverge, in accordance with Eq. (44) for $n=0$, and signalling an extreme form of the WF law's violation.

Now, if the density appearing in Eq. (44) were to be interpreted as its zero-temperature value $n(T=0)$, then in the neutral case the WF ratio would diverge, regardless of the value of $z$. Conversely, if it were to be treated as the equilibrium $T$-dependent density of particles/holes with the dimension given by Eq. (13), then in the neutral system the r.h.s. of Eq. (44) would always be finite, including the case of $z=1$. Clearly, a further clarification on the conditions under which Eq. (44) holds is warranted here.

The scaling analysis of the quantum-critical regime can also be extended to spin dynamics. A small field expansion of the free energy yields the dimension of the spin susceptibility

$$
\left[\chi_{\mathrm{s}}\right]=\left[E / B^{2}\right]=d-\theta+z-4+2 \theta / d \text {. }
$$

However, there seems to be neither a solid holographic result to compare with nor even a commonly accepted recipe for computing this quantity.

The few previous attempts range from using a radial equation for the variation of the vector potential $\delta A_{\perp}$ similar to Eq. (46) in the next Section [57]

$$
\begin{aligned}
& \partial_{r}^{2} \delta A_{\perp}+\partial_{r} \log \frac{\left(\Pi_{i} g_{i i}\left|g_{t t}\right|\right)^{1 / 2}}{g_{r r}^{1 / 2} g_{x x}} \partial_{r} \delta A_{\perp} \\
& -\left(\omega^{2} \frac{g_{r r}}{g_{t t}}+k^{2} \frac{g_{r r}}{g_{y y}}\right) \delta A_{\perp}=0
\end{aligned}
$$

or for the magnetic field itself $\delta B=\partial_{\|} \delta A_{\perp}[58,59]$ to that for the spin connection $\delta \omega_{t}^{x y} \sim \partial_{x} \delta g_{t y}$ [60] from which one can evaluate $\chi_{s}$, as if it was just another response function of the Kubo type.

In particular, in the $2 d$ case and for $\omega / T \gg 1$ the thus-obtained result [58, 59], $\chi_{\mathrm{s}} \sim \omega^{2 / 3}$, was claimed to compare favourably with that experimentally observed in the conjectured spin-liquid state of the quasi- $2 d$ materials $\mathrm{YbRh}_{2}\left(\mathrm{Si}_{1-x} \mathrm{Ge}_{x}\right)_{2}$ and $\mathrm{ZnCu}_{3}(\mathrm{OH})_{6} \mathrm{Cl}_{2}$. It can be easily checked, however, that the scaling dimension (45) does not appear to support the above estimate for any relevant values of $z$ and $\theta$ (see below).

\section{Many shades of holographic conductivity}

Electrical conductivity has been computed for a variety of holographic models and in a number of different ways. However, establishing a consistency between the results of different calculations (or a lack thereof) does not seem to have always been particularly high of the agenda.

The most frequently employed calculation of the electrical conductivity and other kinetic coefficients is based on the holographic adaptation of the Kubo formula [1-4]. It proceeds by solving linearized equations for small variations of the electromagnetic potential $\delta A_{\mu}$ and (possibly) such coupled component(s) of the metric as $\delta g_{t x}$, and/or other degrees of freedom, depending on the field content of the bulk theory in question.

In the case of a generic electro-magneto-gravitational background treated in the customary probe limit by virtue of the DBI action (3), the relevant quasi-normal mode obeys the equation $36-38$. 


$$
\begin{aligned}
& \partial_{r}^{2} \delta A_{i}+\partial_{r} \log \frac{\left(\Pi_{i} g_{i i}\right)^{1 / 2}\left|g_{t t}\right|}{g_{r r}^{1 / 2} g_{x x}^{3 / 2} h\left(k^{2} h^{2}-\omega^{2}\right)} \partial_{r} \delta A_{i}- \\
& -\frac{g_{r r}}{g_{t t}}\left(\omega^{2}-k^{2} h^{2}\right) \delta A_{i}=0,
\end{aligned}
$$

where

$$
h=\sqrt{\frac{\left|g_{t t}\right| g_{r r}-F_{t r}^{2}}{g_{r r} g_{x x}}}=\sqrt{\frac{\left|g_{t t}\right|}{g_{x x}\left(1+(e n)^{2} / \Pi_{i} g_{i i}\right)}} .
$$

As per the standard holographic prescription $1-$

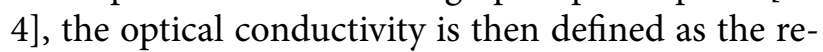
flection coefficient of a radial in-falling wave

$$
\sigma=\left.\operatorname{Im} \frac{r \partial_{r} \delta A_{i}}{\delta A_{i}}\right|_{r \rightarrow 0}
$$

where the Fourier-transformed function $\delta A_{i}(r, \omega, k)$ and its derivative are evaluated at the boundary (a.k.a. the UV limit).

As one technicality, in order to compute Eq. (47) one first solves Eq. (46) in the opposite, IR or $r \rightarrow \infty$, limit (which is also formally attainable by putting $n=0$ ) where it reads

$$
\partial_{r}^{2} \delta A_{i}+(2 \gamma-\alpha+\beta) \frac{\partial_{r} \delta A_{i}}{r}+\omega^{2} r^{2(\alpha-\beta)} \delta A_{i}=0,
$$

the coefficients being those of the metric (4).

Next, imposing the in-falling boundary condition at $r \rightarrow \infty$ one obtains the solution

$$
\delta A_{i} \sim u^{v} H_{v}^{(1)}(u)
$$

where $v=1 / 2-\gamma /(1+\alpha-\beta)$ and $u=\omega r^{z} / z$, which then has to be matched with that of the equation obtained from (46) in the $r \rightarrow 0$ limit by comparing the two in the region $u \sim 1$ where they overlap (which, in turn, requires one to take the small $\omega$ limit).

Skipping the algebra (unlike much-needed physical discussions of the results of this calculation, such formal manipulations, complete with all the auxiliary technical details, can be readily found in many of the pertinent papers), one obtains

$$
\sigma_{\text {Kubo }} \sim \omega^{-2 \gamma /(1+\alpha-\beta)} .
$$

Somewhat surprisingly, instead of deriving this general result once and for all, in much of the holographic literature this calculation would be performed anew for every equation of the type (48).

Also, observe that Eq. (49) is invariant under the transformation (5), in agreement with the aforementioned conformal equivalence of the corresponding metrics.
Barring the fact that under the aforementioned matching condition the power-law dependence (49) is derived for low frequencies, this asymptotic behaviour and its analogues (see below) have been contrasted against the experimental data taken at energies up to $\mathrm{eV}$ (e. g. in the case of the cuprate superconductors [61]).

Applying Eq. (49) to the HV metric (6) paired with the radial electric field (11), one obtains the optical conductivity of a charged $(n \neq 0)$ holographic system

$$
\sigma_{\text {Kubo,charged }} \sim \omega^{-(2 / z)(1-\theta / d)}
$$

for small $\omega$ and $z>2(1-\theta / d)$, while in the opposite case one gets $\sigma \sim 1 / \omega$. This result was reported in Refs. [36-38] (the first two of these references addressed only the limit $z \rightarrow \infty,-\theta / z=$ const, though).

In the neutral case, the conductivity can be obtained by expanding and solving Eq. (46) directly at the boundary $(r \rightarrow 0)$ where the electric field is negligible, thereby yielding

$$
\sigma_{\text {Kubo,neutral }} \sim \omega^{(d-2)(1-\theta / d) / z},
$$

and this estimate is in a perfect agreement with the scaling dimension (25).

In turn, Eq. (50) can then be readily rationalized by observing that the ratio $\sigma_{\text {Kubo,neutral }} / \sigma_{\text {Kubo,charged }}$ scales as the charge density (15), which in the neutral system is played by the density of thermally activated quasiparticles (of either charge sign).

Notably, in the $2 d$ case Eq. (51) allows for no faster than logarithmic dependence. Besides, there seems to be little difference between the general $\mathrm{HV}$ and the Lifshitz $(\theta=0)$ geometries. In that regard, it is worth mentioning that the experimentally measured optical conductivity of a neutral (undoped) $2 d$ Dirac metal, such as graphene, indeed appears to be nearly constant $\left(\sim e^{2} / h\right)$.

Specifically, in Refs. [62-66] the latter was found to behave as

$$
\sigma_{\text {graphene }} \sim \operatorname{Re} \frac{T}{\mathrm{i} \omega+g^{2}(T) T},
$$

where the logarithmically running effective charge $g(T) \sim 1 / \log T$ represents the effect of the Coulomb interactions.

In the higher dimensions $d>2$, according to Eq. (51) the conductivity of a neutral system generally vanishes for $\omega \rightarrow 0$, regardless of the value of $z$, and this behaviour is consistent with the intrinsically semi-metallic nature of such systems. In the pertinent example of the $3 d$ 'Weyl metal' where $z=1$ it was recently found that $\Delta_{\sigma}=3-4 M$ with $|M|<1 / 2[67,68]$. 
It is again instructive to compare Eqs. $(50,51)$ to the predictions of the 'membrane paradigm' which also provides a simple algebraic expression for the low- $\omega$ value of the conductivity. The latter is cast solely in terms of the geometry at the (necessarily, non-degenerate) horizon (thus, such results would not be applicable to the extremal black branes) without the need of solving any diferential equations.

Furthermore, this approach can also be extended to include a magnetic field. To the first order in the weak field $B$, both the diagonal and off-diagonal components of the DC conductivity tensor take the following closed form [53-55]:

$$
\begin{aligned}
& \left.\sigma_{x x} \sim e^{-2 \phi_{0}} \frac{\sqrt{(e n)^{2}+e^{2 \phi_{0}} \Pi_{i} g_{i i}}}{g_{x x}}\right|_{r \rightarrow r_{\mathrm{h}}}, \\
& \left.\sigma_{x y} \sim \frac{e^{-4 \phi_{0}} e n B}{g_{x x} g_{y y}}\right|_{r \rightarrow \mathrm{h}_{\mathrm{h}}},
\end{aligned}
$$

where $\phi_{0}$ is the fixed point value of the dilaton.

Notably, at $\phi_{0}=0$ the first of Eq. (53) reproduces Eqs. (50) and (51) for $n \neq 0$ and $n=0$, respectively, whereas the second one appears to be fully consistent with the scaling dimension (37).

The problem, however, is that, contrary to Eqs. (50) and (51), Eq. (53) is supposed to be evaluated at the horizon, rather than the boundary.

In general, the local conductivity defined according to Eq. (49) at an arbitrary $r$ is expected to be independent of $r$, unless there is a 'running' field, such as an electrical scalar potential or dilaton which can bring about a nontrivial $r$-dependence.

In the neutral case and in the absence of a dilaton, no radial evolution should indeed occur, and so the conductivity could be equally well evaluated either at the boundary or the horizon $\left(\sigma_{\text {Kubo,neutral }}^{(B)}=\sigma_{\text {Kubo,neutral }}^{(H)}\right)$.

However, in the charged case one would expect the local conductivity to vary with the radial variable, whether or not a non-trivial dilaton field is present.

To that end, in Ref. [51] a general relation was proposed

$$
\frac{\sigma^{(B)}}{\sigma^{(H)}}=\left(\frac{S T}{E+P}\right)^{2},
$$

with this ratio becoming unity at zero density, as per the equation of state $(10)$.

In contrast, at finite density Eq. (54) implies a nontrivial radial (hence, temperature) dependence of the local conductivity, thereby predicting the low- $T$ behaviour

$$
\begin{aligned}
& \sigma_{\text {Kubo, charged }}^{(H)} \approx \sigma_{\text {Kubo, charged }}^{(B)}\left(\frac{n \mu}{S T}\right)^{2} \\
& \sim T^{(2 / z)(-d+\theta-z-1+3 \theta / d),}
\end{aligned}
$$

which clearly contradicts Eq. (50).

Conversely, if one chooses to treat Eq. (50) as the horizon value $\sigma_{\text {Kuboneutral }}^{(H)}$, then Eq. (56) implies

$$
\sigma_{\text {Kubo,neutral }}^{(B)} \sim T^{(2 / z)(d-\theta+z-1-\theta / d)},
$$

which is again different from the predictions of the scaling analysis.

This adds to the argument that a better understanding of the applicability of such formulae as Eqs. (44) and (54) for $n \neq 0$ is definitely called for.

However, in spite of some confusion with their terms of use, Eq. (53) can still capture such intrinsic properties of the conductivity tensor as, e. g. the relative scaling of its components with temperature.

Namely, the scaling dimensions (36)-(38) would seemingly imply that the following relation

$$
\sigma_{x y} \sim B \frac{\sigma_{x x}^{2}}{e n}
$$

sets in, as the system approaches the neutral regime at high temperatures. In fact, such a relation does hold - but only for the partial (particle and hole) contributions towards the total Hall conductivity. Should both components happen to have equal mobilities (as, e. g., in the case of a particle-hole symmetric spectrum), the overall $\sigma_{x y}$ would only be proportional to the charge imbalance given by en $(T=0)$, thereby resulting in the different relative scaling rule

$$
\sigma_{x y} \sim B e n(T=0) \frac{\sigma_{x x}^{2}}{(e n)^{2}} .
$$

The naive relation (55) could still hold, though, if the spectrum were lacking particle-hole symmetry (as, e. g., in the case of topological insulators where the Dirac spectrum emerges as a result of the bulk gap inversion).

In that regard, Ref. [49] claimed that in the extended class of the 3-parameter HV systems and at suficiently high temperatures the cuprate-like behaviours of both $\sigma_{x x}$ and $\sigma_{x y}$ could be recovered even in the spatially isotropic case.

Indeed, by using Eq. (56), one can see that in the original, 2-parameter $\mathrm{HV}$ system with $z \neq 0$ and $\theta \neq d$, this does not happen, as the desired dependences $\sigma_{x x} \sim T^{-1}$ and $\sigma_{x y} \sim T^{-3}$ would only occur in the unphysical dimension $d_{\mathrm{s}}=2 / 3$. 
However, in the 3-parameter family of the generalized HV systems, such dependences could emerge under the choice of parameters [49]

$$
\phi=2-3 z / 2, \theta_{\mathrm{m}}=d_{\mathrm{s}}-z / 2,
$$

and these conditions would only be consistent with Eq. (27), provided that

$$
d+2-2 z-\theta=0 .
$$

The tangibility of such a scenario remains to be discerned, as does the whole notion of different dimensions for the gravitational and gauge (matter) degrees of freedom. In the known examples of layered strongly correlated systems, the emergent (both gauge and matter) fields always tend to be confined to the supporting lower-dimensional subspace. In contrast, the physical Coulomb interactions do permeate the surrounding $3 d$ space and their dynamics gets affected by the charges outside the graphene plane, thus hindering the possibility of finding their consistent holographic description.

Yet another important remark is in order here. According to Eq. (53), a finite longitudinal (Ohm's) conductivity can arise due to both current relaxation as well as (Schwinger's) pair production. While the latter mechanism operates even at zero charge density (unlike the current, the system's momentum would then be conserved), the former one requires a finite density of carriers immersed in a dissipative medium composed of some neutral modes.

In the framework of the 'membrane paradigm', the two sources of finite conductivity combine together in a rather peculiar manner

$$
\sigma=\sqrt{\sigma_{\mathrm{mr}}^{2}+\sigma_{\mathrm{pc}}^{2}},
$$

where $\sigma_{\mathrm{mr}}$ and $\sigma_{\mathrm{pc}}$ stand for the contributions due to momentum relaxation and pair creation, respectively.

For comparison, a direct application of the conventional Kubo approach yields [69]

$$
\sigma=\sigma_{\mathrm{mr}}+\sigma_{\mathrm{pc}}
$$

where the two terms can be readily identified as those produced by the inter- and intra-band transitions, respectively.

Notably, both Eqs. (57) and (58) violate the standard Matthiessen's rule, according to which it is the inverse of the partial conductivities (due to different mechanisms of scattering for the same type of carriers) that tend to add up ('connection in series'). In contrast, Eq. (58) presents the combination rule for those situations where there are two different contributions to the overall conductivity in response to the same bias ('parallel connection'), the higher one short-circuiting the rest of the system.

Among the consequences of this unconventional ('anti-Matthiessen') combination rule for the partial scattering rates is a potential dominance of $\sigma_{\mathrm{pc}}$ at high temperatures, resulting in the effective independence of Eqs. (57) and (58) from the momentum relaxation rate in this regime. In that regard, it is worth mentioning the forceful attempt of Ref. [70] to obtain the concomitant cuprate-like behaviours of the conductivity and the Hall angle by speculating that the former behaves as $\sigma \approx \sigma_{\mathrm{pc}} \sim T^{-1}$, whereas the latter acquires the much-desired inverse quadratic dependence, $\theta_{\mathrm{H}} \sim \sigma_{\mathrm{mr}} \sim T^{-2}$ due to the Umklapp processes.

However, this argument appears to ignore the wellknown subtleties of the Umklapp-related phenomena. In particular, no $\sim T^{2}$ momentum-relaxation rate should be expected in the case of a simply-connected $2 d$ convex Fermi surface or a non-Galilean invariant system with an isotropic non-parabolic dispersion (same is also true for an arbitrarily anisotropic parabolic one) [71-73.

Thus, contrary to the naive expectations, the holographic calculations resulting in Eqs. (57) and (58) may need quite a bit of adjusting before their predictions could withstand a comparison with the actual experimental data on the cuprates.

Furthermore, the two partial conductivities in Eqs. (57) and (58) were found to behave as

$$
\sigma_{\mathrm{mr}, 1}(\omega) \sim \omega^{|3+(d-2-\theta) / z|-1}
$$

[74 or

$$
\sigma_{\mathrm{mr}, 2}(\omega) \sim \omega^{|1+(\theta-2-d) / z|-1}
$$

[69], whereas

$$
\sigma_{\mathrm{pc}}(\omega) \sim \omega^{(1-\zeta|\zeta|-1) / z}
$$

[69, 74], where a new ('conduction') exponent $\zeta$ was introduced to describe the scalar potential $A_{0} \sim r^{\zeta-z}$. Conceptually, it can be related to the aforementioned $\phi$-factor.

Given that the exponents appearing in Eqs. (59)(61) differ from those discussed earlier in this Section, a better understanding of their physical nature as well as the origin of combination rules (57) and (58) would once again be warranted.

Interestingly, though, the momentum relaxation exponent (60) found in Ref. [69] agrees with the results of still another recent work of Refs. [75-77] 
where an important effort was made to include elastic scattering, alongside the inelastic one.

In fact, the analysis of Refs. [75-77] represents a 'holography-augmented' transport theory, rather than a systematic all-holographic calculation. Conceivably, though, such a hybrid approach might be better equipped for capturing the underlying physics of the relevant transport phenomena.

Specifically, these works employed the so-called memory function formalism [78-80] which does not explicitly rely on the existence of well-defined quasiparticles and presents, e. g., the electrical conductivity in the form

$$
\sigma_{\text {memory }}=\chi_{J P}^{2}\left(\int \mathrm{d} k k_{x}^{2} \frac{\operatorname{Im} D(\omega, k)}{\omega}-\mathrm{i} \omega \chi_{P P}\right)^{-1},
$$

where $\chi_{I P, P P}(T)$ are the current-momentum and momentum-momentum susceptibilities.

Formula (62) assumes that momentum is the only (nearly) conserved physical quantity and relates the conductivity to the spectral density of the operator that breaks momentum conservation. It is expected to work best in the hydrodynamic regime where the rate of momentum relaxation due to a breaking of translational invariance by elastic impurity or lattice-assisted inelastic Umklapp scattering is smaller than the inelastic rate which controls a formation of the hydrodynamic state itself. For instance, in the case of $\mu \gg T$ the rate of the Umklapp scattering is of order $\sim T^{2} / \mu$, whereas the latter one is given by the universal quantum-critical rate (32).

In general, the onset of hydrodynamics is a distinct property of strong correlations which would be routinely absent in the Fermi liquid regime. Such a regime would also be absent in $1 \mathrm{~d}$, thanks to the peculiar $1 d$ kinematics facilitating the emergence of infinitely many (almost) conserved currents.

In the absence of any (nearly) conserved quantities Eq. (62) ceases to be applicable. Although the corresponding 'incoherent' metals do not allow for any simple description, they have been eloquently argued [81-83] to conform to the ubiquitous $\sigma \sim 1 / T$ dependence stemming from the universal scattering rate (32).

In many cases, though, strong interactions often go hand-in-glove with (and enhance the effects of) strong disorder. The combined effects of the two can hardly be accounted for by means of the perturbative AltshulerAronov theory and are likely to require some intrinsically non-perturbative approaches, such as the EfrosShklovskii one, thus allowing for other, essentially non-linear, $T$-dependences, $\sigma \sim \exp \left(-\# / T^{\alpha}\right)$.

It is also worth noting that, unlike Eqs. (57) and (58), the applications of Eq. (62) would have a good chance to be in compliance with the Matthiessen's rule, as different scattering mechanisms tend to correspond to separate contributions to the integral kernel $D(\omega, k)$, thereby producing additive terms in the expression for the inverse conductivity.

The main inference from Eq. (62) is a transfer of the spectral weight from the coherent Drude peak to the incoherent high-frequency tail. It is worth noting, though, that in the previous applications of Eq. (62) a possible quasiparticle renormalization was not, de facto, considered, as the behaviour of $\chi_{I P, P P}$ was believed to be non-singular and, at most, only weakly $T$-dependent (such an assumption notwithstanding, e. g., at the onset of the Mott transition one expects $\left.\chi_{J P}=0\right)$.

As mentioned above, the behaviour found in Refs. 75-77

$$
\sigma_{\text {memory }} \sim T^{(\theta-2-d) / z}
$$

coincides with that reported in Ref. [69]. However, in Refs. [75-77] it was shown to emerge only in the strong coupling regime, whereas the lowest (second) order perturbative result was found to be nonuniversal $\sigma_{\text {memory }} \sim T^{(z-d-\delta) / z}, \delta$ being the anomalous dimension of the operator that breaks momentum conservation.

Taking into account the HV scaling relations (16) one observes that in the neutral case Eq. (63) appears to be inversely proportional to entropy (equivalently, speci_cheat or viscosity), as conjectured earlier in Ref. [52]. However, the dependence $\sigma \sim 1 / S_{\text {ent }} \sim T^{(\theta-d) / z}$ advocated in [52] can only occur in the limit $z \rightarrow \infty$. Otherwise, Eq. (63) features an additional factor that, incidentally, behaves as the inverse square of a $T$-dependent 'graviton mass' $m \sim T^{1 / z}$.

In a series of works [84-89], it was indeed proposed to incorporate the effects of static disorder by introducing a graviton mass $m$ which is weakly (if at all) $T$-dependent. Although under such an assumption the desired dependence $\sigma \sim 1 / S_{\text {ent }}$ does indeed set in, it remains to be seen whether such a scenario can be justified beyond the ad hoc level.

As yet another effort towards marrying the formal holographic manipulations with the more traditional transport theory, it was also proposed to mimic the momentum-relaxing Umklapp processes brought about by the presence of a regular crystal lattice with expressly anisotropic geometries and periodic scalar and/or dilaton potentials.

To that end, in the previously quoted Refs. [10-13] the crystal lattice was modelled by a periodic electric potential, resulting in $\Delta_{\sigma}=-2 / 3,-\sqrt{3} / 2$ in $2 d$ and $3 d$, respectively. 
Also, noteworthy is the proposal [90] to use the helical Bianchi-VII ${ }_{0}$ metric with a pitch in the $x$ direction

$$
\begin{aligned}
& g_{t t}=-g_{r r} \sim-1 / r^{2}, g_{x x} \sim r^{2 / 3}, \\
& g_{y y} \sim 1 / r^{4 / 3}, g_{z z} \sim 1 / r^{2 / 3}
\end{aligned}
$$

as a holographic description of the anisotropic $3 d$ periodic structure which gives rise to the interaction-induced Mott-type state with the 'bad-metallic' conductivity $\sigma_{\mathrm{xx}} \sim T^{4 / 3}$ in the direction of the pitch, alongside a gapless behaviour of the entropy, $S \sim T^{2 / 3}$.

Although the Bianchi geometry (64) does have its intellectual appeal, it should be noted that the above choice is not unique. As follows from Eq. (51), the same behaviour of both $\sigma_{x x}$ and entanglement entropy can be found for an entire family of the uniaxially anisotropic $3 d$ metrics

$$
g_{t t} \sim-1 / r^{2 \alpha}, g_{r r} \sim 1 / r^{2 \beta}, g_{i i} \sim 1 / r^{2 \gamma i}
$$

which satisfy the conditions $2 \gamma_{x} /(1+\alpha-\beta)=-2 / 3$, $\Sigma_{i} \gamma_{i}=2 / 3$. For instance, by choosing $\alpha=\beta=1$; $\gamma_{x}=-\gamma_{y}=-\gamma_{z}=-2 / 3$ one finds that fully spatially anisotropic geometries, as in (64), may not be necessary for constructing a holographic dual of the 'bad metal', after all.

Continuing with the list of the previously obtained holographic results it might be worth mentioning a few more examples whose physical interpretation (as well as mutual consistency) is yet to be ascertained.

For one, there has been a variety of predictions for the dimension of electrical conductivity. In the neutral Lifshitz case $(\theta=0)$, Refs. [44-48] found $\Delta_{\sigma}=$ $(d+2-2 z) / z$, while Ref. [91] reported $\Delta_{\sigma}=(3-d-z) / z$, and Ref. [92] arrived at the exponent $\Delta_{\sigma}=(d+2 z-4) / z$.

None of these values appears to be consistent with the above scaling predictions and the universal quantum-critical scattering rate (32).

Going beyond the Lifshitz case, Ref. [93] found $\Delta_{\sigma}=3$ for $d=3$ and $\Delta_{\sigma}=(2 z-3) / z$ for $\theta=d-1$, whereas Ref. [94] reported $\Delta_{\sigma}=(d-2) / z$, but only for $z=d-2$, $\Delta_{\sigma}=1 / z$ for $z=(d-4) / 3$, and $\Delta_{\sigma}=(d-\theta) / z$, also in conflict with the above scaling results.

Also, for $d=2$ Ref. 951 delivered $\Delta_{\sigma}=2$, while Ref. [96] obtained $\Delta_{\sigma}=7 / 2$. Moreover, Ref. [97] presented an even greater variety of values, $\Delta_{\sigma}=1,2,3, d$, $d-2, d-4$ for $z=1$ and $(2 z+d-2) / z$ for $z \neq 1$, as well as a whole discrete series $(1+3 p) /(3+p)$, whereas other works featured the entire plethoras of non-universal exponents as functions of one or even two continuous parameters appearing in the holographic Lagrangian [98, 99].
On the other hand, Refs. [100-102] utilized the metrics (4) with $\beta=2-\alpha$ obtaining the results $\Delta_{\sigma}=-2 \gamma /$ $(2 \alpha-1)$ and $\cot \theta_{\mathrm{H}} \sim T^{2 \gamma /(2 \alpha-1)}$, in agreement with (49).

Still other available methods of computing conductivity include extracting it from the hydrodynamic expansion or computing a drag force for massive charge carriers. Although some of those results may seem more plausible than others, they are still awaiting for their physical interpretation and a systematic comparison with the predictions made by the alternative techniques.

In that regard, the general universal relations, such as Eqs. (28) or (33), provide an important consistency test, while reinforcing the notion that the dynamic properties of quantum-critical systems are closely related to their thermodynamics. Technically, such a relationship implies that, apart from the relaxation rate (32), the kinetic coefficients can be found in terms of the thermodynamic ones.

Yet another important test would be provided by the sum rules for the optical conductivity and other kinetic coefficients, akin to those extensively employed in Refs. [103, 104]. Obviously, no monotonic low-frequency asymptotic obtained by solving the differential equation (46) in the Kubo formula approach can be up for this test. However, the frequency-dependent counterparts of the purely algebraic Eqs. (53) could indeed be used to that effect, once their closed expressions are obtained in a wide range of frequencies.

\section{Mother of all non-Fermi liquids}

One concrete context for a comparative discussion of different holographic models is provided in the theories of fermions coupled to gapless over-damped bosonic modes. This 'mother of all NFLs' has long been at the forefront of theoretical research, since the singular interactions mediated by soft gauge field-like bosons are often associated with incipient ground state instabilities and concomitant NFL types of behaviour.

Such effective long-range and strongly retarded interactions may occur even in microscopic systems with purely short-ranged couplings. In the close proximity to a quantum-critical point, the role of the corresponding modes is then played by (nearly) gapless excitations of an emergent order parameter.

Important examples include such problems as ordinary electromagnetic fluctuations in metals and plasmas, spin and charge ordering transitions in itinerant (anti)ferromagnets, compressible Quantum Hall Effect, Pomeranchuk instabilities resulting in rotationally anisotropic 'quantum nematic' states, etc. 
Despite all the differences in their physical nature, these systems conform to the general problem of a finite density fermion gas coupled to an overdamped bosonic mode whose own dynamics is governed by the (transverse) gauge field-like propagator:

$$
D(\omega, q)=\frac{1}{|\omega| / q^{\xi}+q^{\rho}} .
$$

In the context of electrodynamics of conducting media, the first and second terms account for the effects of the Landau damping and diamagnetism, respectively.

Over the past two decades this problem has been repeatedly attacked with a variety of techniques. At the early stage, it was believed that the functional form of the one-loop fermion self-energy

$$
\Sigma(\omega)=\int \mathrm{d} \epsilon \mathrm{d}^{d} q \frac{D(\omega, q)}{(\mathrm{i} \omega+\mathrm{i} \epsilon-\mathbf{v q})} \sim \omega^{\frac{d-1+\xi}{\xi+p}}
$$

survives in the higher orders of the perturbation theory, akin to the situation in the Eliashberg theory of electron-phonon interactions [105, 106. However, the more recent analyses demonstrated an inapplicability of the naive weak coupling and $1 / N$ expansions [107-109], thus calling the earlier results into question.

There have also been attempts to study this theory without introducing the Landau damping from the outset [110, 111], and such analysis yields a selfenergy $\Sigma \sim \omega^{1-\epsilon / 4}$ in $d=3-\epsilon$ dimensions and for $\rho=2$, $\xi=1$ that is markedly different from the counterpart of (67), $\Sigma \sim \omega^{1-\epsilon / 3}$. In still other approaches, the problem was attacked by expanding in $z_{\mathrm{b}}-2$ [112] or $d=5 / 2-\epsilon[113]$.

Despite somewhat conflicting results, Eqs. (66) and (67) would often be used for evaluating the boson and fermion dynamical exponents

$$
z_{\mathrm{b}}=\rho+\xi, z_{\mathrm{f}}=\frac{\xi+\rho}{d-1+\xi} .
$$

Conceivably, a hypothetical holographic dual (if any) of the boundary theory with the interaction (66) might involve such bulk degrees of freedom as gauge potential, metric and scalar fields and, therefore, it could be envisioned amongst the solutions of the EMD Lagrangian (9).

Along these lines, in Ref. [114] a comparison was made between the two-point correlation function computed holographically in a yet-to-be-specified HV geometry and those obtained directly in the boundary theory with the use of the eikonal technique.
The agreement was found, provided that the $\theta$-parameter of the HV metric (6) was chosen as

$$
\theta=d \frac{\rho+1-d}{\xi+d-1}
$$

thereby satisfying the relation $z_{\mathrm{f}}=1+\theta / d$ and, incidentally, turning the first of the conditions (8) into an exact equality. In particular, for $d=2, \rho=2, \xi=1$ one obtains $z_{\mathrm{f}}=3 / 2$ and $\theta=1$, the values that have also been independently singled out on the basis of analysing the entanglement entropy [41, 42].

Table. Comparison of the exponents governing a powerlaw decay of the conductivity computed holographically.

\begin{tabular}{c|c|c|c}
\hline$\Delta_{\sigma}$ & Kubo $_{\text {charged }}$ & Kubo $_{\text {neutral }}$ & $\begin{array}{c}\text { Memory } \\
\text { function }\end{array}$ \\
\hline $\begin{array}{c}\text { Model } \\
\text { I }\end{array}$ & $\frac{-2}{d z_{\mathrm{f}}}$ & $\frac{d-2}{z_{\mathrm{f}} d}$ & $\frac{-3}{z_{\mathrm{f}}}$ \\
\hline $\begin{array}{c}\text { Model } \\
\text { II }\end{array}$ & $\frac{-2}{z_{\mathrm{b}}}$ & $\frac{d-2}{z_{\mathrm{b}}}$ & $\frac{-(d+2)}{z_{\mathrm{b}}}$ \\
\hline $\begin{array}{c}\text { Model } \\
\text { III }\end{array}$ & $\frac{-2}{z_{\mathrm{b}}}$ & $\frac{d-2}{z_{\mathrm{b}}}$ & $-\frac{d}{z_{\mathrm{b}}}-\frac{2}{z_{\mathrm{f}}}$ \\
\hline $\begin{array}{c}\text { Model } \\
\text { IV }\end{array}$ & $\frac{-2}{z_{\mathrm{b}} d}$ & $\frac{d-2}{z_{\mathrm{f}} d}$ & $-\frac{1}{z_{\mathrm{f}}}-\frac{2}{z_{\mathrm{b}}}$ \\
\hline $\begin{array}{c}\text { Model } \\
\text { V }\end{array}$ & $2-\frac{4}{z_{\mathrm{f}}}$ & $2-d+\frac{2(d-2)}{z_{\mathrm{f}}}$ & $d-\frac{2(d+1)}{z_{\mathrm{f}}}$ \\
\hline
\end{tabular}

In the Table, we compare the exponents governing a power-law decay of the conductivity computed holographically with the use of Eqs. (50), (51), and (62). These values pertain to the aforementioned models IIV and are complemented by those for the new Model $\mathrm{V}$ which is characterized by the exponents $z_{\mathrm{f}}$ and $\theta$ given by Eqs. (68) and (69), respectively.

The first two columns contain the exponents $\Delta_{\sigma}^{(\infty)}$ governing the $\omega$-dependence for $\omega \gg T$ and given by Eqs. (50) and (51), whereas the third column contains the values of $\Delta_{\sigma}^{(0)}$ pertinent to the $T$-dependence for $\omega \gg T$ and given by Eq. (62) (a potentially strong $\omega$-dependence of the functions $\chi_{I P, P P}$ complicates the analysis of $\sigma(\omega)$ in the framework of the memory function method).

A few comments are in order.

Firstly, despite being spurious as far as its physical implications are concerned (see below), the muchdesired exponent $-2 / 3$ is quite robust and can be obtained in any of the models I-IV for both $d=2$ and $d=3$, as long as $\xi=1$. Moreover, for $\xi=1$ all the results for the models I and III as well as those for the models II and IV are identical. 
Secondly, the Model V with its conjectured boundary dual represented by the gauge-fermion model can also be amenable to the application of the standard Drude theory. The latter (nominally) assumes the existence of a quasiparticle description and yields the conductivity

$$
\sigma_{\text {Drude }} \sim \operatorname{Re} \frac{1}{\mathrm{i} \omega / Z+\Gamma_{\text {tr }}},
$$

where the (possibly strong) quasiparticle renormalization is accounted for via the Green function's residue, $Z=1 /(1-\partial \Sigma / \partial \omega)$.

Potentially, Eq. (70) can take rather different forms at small and large $\omega$, as compared to $T$, depending on whether or not the transport scattering rate $\Gamma_{\text {tr }}$ behaves differently from that of quasiparticle decay.

Estimating the latter as $\Sigma(\omega) \sim \omega^{1 / z_{\mathrm{f}}}$ one then finds the former (as well as the entire $T$-dependent DC conductivity) to be governed by the modified exponent

$$
\sigma_{\text {Drude }}^{(0)} \sim 1 / \Gamma_{\mathrm{tr}} \sim T^{-\left(1 / \mathrm{z}_{\mathrm{f}}+2 / z_{\mathrm{b}}\right)} .
$$

In contrast, at high $\omega$ one obtains

$$
\sigma_{\text {Drude }}^{(\infty)} \sim \frac{Z^{2} \Gamma_{\text {tr }}}{\omega^{2}} \sim \omega^{-1 / z_{\mathrm{f}}+2 / z_{\mathrm{b}}} .
$$

Thus, the DC and AC Drude conductivities exhibit the same exponent only in the limit $z_{\mathrm{b}} \rightarrow \infty$ which corresponds to a particular case of the generic short-ranged (and, therefore, only weakly momentum-dependent) scattering mechanism.

By contrast, for any finite $z_{\mathrm{b}}$ there will be a disparity between the transport and quasiparticle decay rates and, as a result, different values of the exponents controlling the $\omega$ and $T$ dependences. This fairly mundane observation should be contrasted with such exotic proposals as a 'wrong' sign of the expression under the absolute value in Eqs. (59)-(61) or a parameter-dependent dominance of one term in Eq. (58) over the other which were put forward in Ref. [69].

By applying Eq. (70) to the Model V one observes that the low- $\omega$ Drude conductivity (specifically, its exponent $\Delta_{\sigma}^{(0)}$ ) agrees with that of the memory function approach for, at least, one of the models I-IV, while in the high- $\omega$ regime (i. e., for $\Delta_{\sigma}^{(\infty)}$ ) this is generally not the case.

Specifically, for $d=2, \rho=2, \xi=1$ the high- $\omega$ Drude formula yields $\Delta_{\sigma}^{(\infty)}=0$, while in $3 d$ one gets $\Delta_{\sigma}^{(\infty)}=-1 / 3$. By contrast, in the DC limit one gets $\Delta_{\sigma}^{(0)}=-4 / 3$ and $-5 / 3$ (up to a power of logarithm) in $2 d$ and $3 d$, respectively.

In the case of $\rho=2, \xi=0$, the counterparts of the above values read $\Delta_{\sigma}^{(\infty)}=1 / 2(2 d)$ and 0 in $(3 d)$, while in the DC limit one gets $\Delta_{\sigma}^{(0)}=-3 / 2$ and -2 in $2 d$ and $3 d$, correspondingly.

The latter estimate should not be compared directly with the prediction $\Delta_{\sigma}^{(\infty)}=-1 / 3$ made in Refs. [115, 116] for the scenario of an incipient $2 d$ spin density wave instability with the large momentum. The cuprate-like shape of the Fermi surface and the dominant scattering involving its opposite regions modify the above results obtained under the assumption of a spherical Fermi surface by a missing factor of $\omega^{2 / z_{b}}$ due to the scattering between the conjugate pairs of hot spots and an additional factor of $\omega^{1 / 2}$ due to a finite span of the region around each hot spot. Together, the two effects conspire to result in a somewhat accidental cancellation, thereby producing $\Delta_{\sigma}^{(\infty)}=0$.

As to the quoted exponent $-1 / 3$, it was obtained in 115, 116 by going well beyond the Drude approximation and focusing on certain 'energy transfer' processes which involve pairs of soft bosons with small total momenta.

The story does not seem to end there, though. The calculation performed in the framework of the 'Ising nematic' model focusing on the (quasi)static fluctuations of the nematic order parameter produced the low- $T$ behaviour [75-77]

$$
\sigma_{\mathrm{IN}} \sim(T \log T)^{1 / 2} .
$$

Under a closer inspection, Eq. (73) turns out to be indicative of the IR divergence of the momentum integral in Eq. (62) from which it is rescued by introducing a cut-off at energies of the order of the mass of the bosonic mode $m \sim(T \log T)^{1 / 2}$.

However, should such a mass happen to be prohibited on the grounds of, e. g., unbroken gauge invariance, the problem in question would turn out to be intrinsically strongly-coupled and possibly resulting in quite different, yet to be determined, conductivity behaviour (in the case of the Ising nematic, such a possibility was claimed to be conveniently preempted by the onset of a super-conducting instability [117).

Besides, albeit being seemingly innocuous to the first order [75-77], the $T$-dependent corrections to the susceptibilities $\chi_{I P, P P}$ may get promoted to the exponent in the higher orders, thus altering the overall power counting.

In that regard, a particularly interesting would be the actual gauge field problem where, unlike the longitudinal, the transverse gauge boson does not develop any (thermal) mass, except in the case of a symmetrybreaking phase transition.

Finally, as a general caveat, the naive scaling predictions for the exponents controlling the temperature 
dependence of conductivity may not be at all observable. This can happen when the prefactor in front of the corresponding term vanishes, as per the analysis based on the semiclassical kinetic equation which was also argued to remain applicable even beyond the Fermi liquid regime [71-73].

\section{Real-life non-Fermi liquids}

The list of documented NFLs is extensive and includes ferromagnetic metals (e. g., $\mathrm{MnSi}, \mathrm{ZrZn}_{2}$ ) and superconductors ( $\left.\mathrm{UGe}_{2}, \mathrm{URhGe}, \mathrm{UCoGe}\right)$, heavy fermions (e. g., $\mathrm{YbRh}_{2} \mathrm{Si}_{2}, \mathrm{CeCoIn}_{5}$ or $\mathrm{URu}_{2} \mathrm{Si}_{2}$ ), unconventional superconductors such as cuprates and iron pnictides, electronic nematics (e. g., $\mathrm{Sr}_{3} \mathrm{Ru}_{2} \mathrm{O}_{7}$ ), insulating magnets (e. g., $\mathrm{CoNb}_{2} \mathrm{O}_{6}$ and $\mathrm{TlCuCl}_{3}$ ), quasi-one dimensional Mott insulators (e. g., (TMTSF) ${ }_{2} \mathrm{PF}_{6}$ or (TMTSF) ${ }_{2} \mathrm{ClO}_{4}$ ), etc.

Given that in most cases the dynamical exponent $z>1$, one might naively expect all the $3 d$ systems to show the classical mean-field scaling behaviour, since the effective dimension of spatial fluctuations, which equals $d+z$, appears to exceed the upper critical dimension $d_{\mathrm{uc}}=4$.

Moreover, the FM systems with a conserved order parameter and $z_{\mathrm{b}}=\rho+\xi=3$ would be anticipated to follow the classical scenario for any $d>1$, whereas for the AFM ones (where the order parameter is not conserved and $z_{\mathrm{b}}=\rho+\xi=2$ ) it would then happen for all $d>2$.

However, this argument can be invalidated by dangerously irrelevant variables presenting a potential source of hyperscaling violation and resulting in the breakdown of the corresponding relation between the specific heat $(\alpha)$ and correlation length $(v)$ exponents, $d v=2-\alpha[118]$.

For one, the $3 d$ helical ferromagnet $\mathrm{MnSi}$ demonstrates NFL behaviour for $\sigma \sim T^{-3 / 2}$ (which reverts to $\sim T^{2}$ in a field of $3 \mathrm{~T}$ ). The itinerant ferromagnet $\mathrm{ZrZn}_{2}$ shows somewhat similar properties. Such prototypical NFL materials have long been viewed as potential candidates to the application of the $3 d$ gauge-fermion theory discussed in the previous Section, although the thus-obtained conductivity would behave as $\sim T^{-5 / 3}$, in disagreement with the above dependences.

In turn, the $3 d$ AFM heavy-fermion compound $\mathrm{YbRh}_{2} \mathrm{Si}_{2}$ exhibits a quantum-critical point at a finite field $H_{\mathrm{c}}$, featuring $\chi_{\mathrm{s}} \sim T^{1 / 4} ; C \sim \mathrm{T}^{3 / 4} ; \sigma \sim T^{-3 / 4}$, the behaviour that is suggestive of the critical exponents $z=4$, $\alpha=1 / 4$, and $v=1 / 3$.

Its doped cousin $\mathrm{YbRh}_{2}\left(\mathrm{Si}_{1-x} \mathrm{Ge}_{\mathrm{x}}\right)_{2}$ shows a power-law behaviour of the low- $\omega$ spin susceptibility $\operatorname{Re} \chi_{\mathrm{s}} \sim T^{-0.6}, \operatorname{Im} \chi_{\mathrm{s}} \sim \omega / T^{1.6}$ for $x \approx 0.05$ 119.
Another example of the $3 d$ AFM materials, $\mathrm{UCu}_{5-x} \mathrm{Pd}_{x^{\prime}}$ manifests $C \sim T^{2}, \sigma \sim T^{-1 / 3}$ and $\chi_{\mathrm{s}} \sim T^{\gamma}$, where $\gamma$ ranges between 0 for $x=1$ (i. e., $\chi_{\mathrm{s}} \sim \ln T$ ) and $\chi_{\mathrm{s}} \sim T^{-1 / 3}$ for $x=1.5$ [118].

The list of the $3 d$ AFM also includes CeIn $_{3}$ with $\sigma \sim T^{-3 / 2}$ (under near-critical pressure), $\mathrm{CePd}_{2} \mathrm{Si}_{2}$ with $\sigma \sim T^{-5 / 4}$, CeRnSn with anisotropic resistivity: $\sigma_{a b} \sim T^{-3 / 2}$, $\sigma_{c} \sim 1 / T$, and magnetic susceptibility: $\chi_{\mathrm{s}, a b} \sim T^{-1 / 3}$, $\chi_{\mathrm{s}, c} \sim T^{-1.5}$.

Another (this time, quasi-2d) AFM material, $\mathrm{CeCu}_{6-x} \mathrm{Au}_{x^{\prime}}$, shows $C \sim T^{7 / 8}, \delta_{\mathrm{s}} \sim T^{-7 / 8} ; \chi_{\mathrm{s}} \sim T^{1 / 8}$ for $x \approx 0.1$ [120], data hinting at the exponents $z=8 / 3, \alpha=1 / 8$, $v=3 / 7$.

It was also argued in Ref. [118] that such data could be explained in terms of the anisotropic dynamical susceptibility $\chi_{s}(\omega, k)=\left(k^{2}{ }_{\perp}+k_{\|}{ }^{4}+|\omega|^{\gamma}\right)^{-1}$ where $\gamma=4 / 5$, yielding $\rho \sim T, \chi_{\mathrm{s}} \sim T^{1 / 2}$.

As regards the quasi-2d AFM materials, the Kagome $\mathrm{AFM} \mathrm{ZnCu}_{3}(\mathrm{OH})_{6} \mathrm{Cl}_{2}$ (a.k.a. Herbertsmithite) shows a power-law behaviour of the bulk susceptibility, $\chi_{\mathrm{s}} \sim T^{-2 / 3}$, and the spin relaxation rate $1 / T_{1} \sim T^{0.7}$, although the issue of its possibly non-analytical $\omega$-dependence at small $\omega$ has not been completely settled yet [121].

Also, the in-plane optical conductivity of this material $\sigma \sim \omega^{7 / 5}$ was argued to be consistent with the picture of a spin gapless (since $C \sim T$ ) but charge gapped $2 d$ Dirac spin liquid state [122].

The gauge theory of the $U(1)$ spin-liquid states is also expected to reproduce such observed metal-like properties as $C / T, \chi_{\mathrm{s}}, \kappa / T \rightarrow$ const (despite $1 / T_{1} \sim T^{2}$ which might indicate a soft nodal gap) in the organic compound $\mathrm{EtMe}_{3} \mathrm{Sb}\left[\mathrm{Pb}(\mathrm{dmit})_{2}\right]_{2}$ which shows the conductivity exponent $\Delta_{\sigma}$ varying between $3 / 4$ to $3 / 2$ [123]. A similar spin-liquid state (although, possibly, with a small spinon gap) characterized by the conductivity exponent $\Delta_{\sigma}$ ranging between 0.8 and 1.5 occurs in $\kappa$-BEET- $\mathrm{Cu}_{2} \mathrm{CN}_{3}$ [124]).

While the complete theory is still being developed and perfected, its viable variant was proposed in the framework of the phenomenological analysis of Ref. [125] where the NFL self-energy was assumed to be independent of momentum, $\Sigma(\omega) \sim \omega^{1-\alpha}$, and subject to the self-consistent equation

$$
\Sigma(\omega)=\int \mathrm{d} \epsilon d^{d} p \Lambda^{2}(\epsilon) \chi_{\mathrm{E}}(\omega+\epsilon, p) G(\epsilon, p),
$$

where $G(\omega, q)=(\mathrm{i} \omega / Z-\mathbf{v q})^{-1}$ is the fermion Green function and $\chi_{\mathrm{E}}(\omega, q)=\int d \epsilon \mathrm{d}^{d} p \Lambda^{4} G G D D$ is the effective propagator of soft bosonic pair-exchange processes, each of which is described by the antiferromagnetic dynamical susceptibility $\chi(\omega, q)$. The latter conforms to the general Eq. (66) where $\xi=0, \rho=2$, and $\omega$ is multiplied by the factor $\Lambda^{2}$ in order to achieve self-consistency. In turn, the interaction vertices are decorated with 
the renormalization factor, $\Lambda \sim 1 / Z$, thereby enforcing the corresponding Ward identity.

The exponents obtained by solving Eq. (74) turn out to be completely universal and solely determined by the spatial dimension

$$
\alpha=1 / 2-1 / z_{\mathrm{b}}, v=\frac{1}{2+z_{\mathrm{b}} \alpha}, z_{\mathrm{b}}=4 d / 3, z_{\mathrm{f}}=\frac{1}{1-\alpha} .
$$

The resulting observables

$$
C \sim T^{1-\alpha}, \sigma \sim \omega^{\alpha-1}, \chi_{s} \sim T^{\alpha}
$$

turn out to describe quite well the aforementioned data on $\mathrm{YbRh}_{2} \mathrm{Si}_{2}$ and $\mathrm{CeCu}_{6-x} \mathrm{Au}_{x}$ for $d=3$ and 2, respectively.

Despite being ostensibly universal, the above solution can only emerge, provided that the initial value of $Z$ is suficiently small [125]. This way, one can explain the fact that this solution does not apparently describe all the antiferromagnetic heavy fermion materials. Nonetheless, it would be a real challenge (and an impressive achievement in the case of success) for the holographic approach to reproduce more than one of the above exponents (for the same compound).

\section{Summary}

The holographic approach aspires to provide a potential framework for treating those strongly coupled systems that do not fit into the conventional quasiparticle picture but could be still amenable to a description in terms of certain one- and two-particle Green functions. In fact, had this implicit assumption failed as well, it would make any comparison with the experimental data (deduced by means of the available oneand two-particle probes) rather problematic.

To that end, a comparison with the results obtained by other, more traditional, techniques might be helpful for setting up a proper holographic model. Besides, in order to become a viable practical tool, the holographic approach would have to be able to reproduce the behaviour of not just one, but a whole variety of observables, such as specific heat, compressibility, magnetic susceptibility, electrical, thermal, and spin conductivities, etc. A host of such data on the documented NFL materials is available and, for the most part, is still awaiting its interpretation.

In the present communication, a number of the existing holographic predictions for thermodynamic and kinetic coefficients in the theories dual to the HV geometries were analysed in the framework of the scaling theory and with an eye on the general universal relations. In the course of such analysis, many striking contradictions and mutual inconsistencies between the predictions for, e. g., the conductivity obtained by virtue of the Kubo vs 'membrane paradigm' techniques (by the same and/or different authors), were exposed and the related subtleties emphasized.

To summarize, providing a solid physical interpretation of the holographic results should be instrumental for ascertaining their true status. In the absence of such physical input, the only (obviously, unwanted) alternative for the holographic predictions would be to get stuck in the situation where any formal result would seem to be (almost) as good as any other one. Only after having proven to be more than tenuously related to the actual materials, the holographic approach will become a genuine breakthrough in the field of strongly correlated systems.

\section{Acknowledgements}

The author acknowledges the hospitality at the Aspen Center for Physics funded by the NSF under Grant 1066293 and at the International Institute of Physics in Natal, Brazil, funded by MCTI and MEC.

\section{References}

[1] S.A. Hartnoll, Classical Quant. Grav. 26, 224002 (2009), http://dx.doi.org/10.1088/0264$9381 / 26 / 22 / 224002$

[2] C.P. Herzog, J. Phys. A 42, 343001 (2009), http:// dx.doi.org/10.1088/1751-8113/42/34/343001

[3] J. McGreevy, Adv. High Energ. Phys. 2010, 723105 (2010), http://dx.doi.org/10.1155/2010/723105

[4] S. Sachdev, Annu. Rev. Cond. Matter Phys. 3, 9 (2012), http://dx.doi.org/10.1146/annurev-conmatphys-020911-125141

[5] W. Witczak-Krempa, E. Sorensen, S. Sachdev, Nature Phys. 10, 361 (2014), http://dx.doi.org/10.1038/ nphys2913

[6] W. Witczak-Krempa, Phys. Rev. B 89, 161114 (2014), http://dx.doi.org/10.1103/PhysRevB.89.161114

[7] S. Bai and D.-W. Pang, arXiv:1312.3351

[8] E. Katz et al., arXiv:1409.3841

[9] K. Chen et al., Phys. Rev. Lett. 112, 030402 (2013), http://dx.doi.org/10.1103/PhysRevLett.112.030402

[10] G.T. Horowitz, J.E. Santos, and D. Tong, arXiv: 1204.0519

[11] G.T. Horowitz, J.E. Santos, and D. Tong, arXiv: 1209.1098

[12]Yi Ling et al., J. High Energ. Phys. 11, 006 (2013), http://dx.doi.org/10.1007/JHEP11(2013)006

[13] D. Vegh, arXiv:1301.0537

[14] A. Donos and J.P. Gauntlett, arXiv:1311.3292

[15]A. Donos and S.A. Hartnoll, Phys. Rev. D 86, 124046 (2012), http://dx.doi.org/10.1103/ PhysRevD.86.124046 
[16]T. Faulkner et al., Phys. Rev. D 88, 045016 (2013), http://dx.doi.org/10.1103/PhysRevD.88.045016

[17]N. Iqbal and H. Liu, Fortsch. Phys. 57, 367 (2009), http://dx.doi.org/10.1002/prop.200900057

[18] M. Cubrovic, J. Zaanen, and K. Schalm, Science 325, 439 (2009), http://dx.doi.org/10.1126/science.1174962

[19] M. Cubrovic, J. Zaanen, and K. Schalm, arXiv: 1012.5681

[20]S.S. Lee, Phys. Rev. D 79, 086006 (2009), http:// dx.doi.org/10.1103/PhysRevD.79.086006

[21] H. Liu, J. McGreevy, and D. Vegh, Phys. Rev. D 83, 065029 (2011), http://dx.doi.org/10.1103/ PhysRevD.83.065029

[22]T. Faulkner et al., Phys. Rev. D 83, 065029 (2011), http://dx.doi.org/10.1103/PhysRevD.83.125002

[23] T. Faulkner and J. Polchinski, J. High Energ. Phys. 6, 012 (2011), http://dx.doi.org/10.1007/JHEP06(2011)012

[24] S. Kachru, X. Liu, and M. Mulligan, Phys. Rev. D 78, 106005 (2008), http://dx.doi.org/10.1103/ PhysRevD.78.106005

[25] C. Charmousis et al., J. High Energ. Phys. 11, 151 (2010), http://dx.doi.org/10.1007/JHEP11(2010)151

[26]B. Goutéraux et al., J. High Energ. Phys. 1,089(2012), http://dx.doi.org/10.1007/JHEP01(2012)089

[27]B. Goutéraux and E. Kiritsis, J. High Energ. Phys. 12, 036 (2011), http://dx.doi.org/10.1007/ JHEP12(2011)036

[28] N. Iizuka et al., arXiv:1105.1162

[29]Xi Dong et al., J. High Energ. Phys. 6, 041 (2012), http://dx.doi.org/10.1007/JHEP06(2012)041

[30] E. Perlmutter, arXiv:1205.0242

[31]C. Charmousis, B. Goutéraux, and E. Kiritsis, I. High Energ. Phys. 09, 011 (2012), http://dx.doi. org/10.1007/JHEP09(2012)011

[32] B. Goutéraux and E. Kiritsis, J. High Energ. Phys. 4, 053 (2013), http://dx.doi.org/10.1007/JHEP04(2013)053

[33]J. Gath et al., J. High Energ. Phys. 4, 159 (2013), http://dx.doi.org/10.1007/JHEP04(2013)159

[34] S.A. Hartnoll and A. Tavanfar, Phys. Rev. D 83, 046003 (2011), http://dx.doi.org/10.1103/PhysRevD.83.046003

[35] S.A. Hartnoll, D.M. Hofman, and D. Vegh, arXiv: 1105.3197

[36]D.-W. Pang, Phys. Rev. D 88, 046002 (2013), http:// dx.doi.org/10.1103/PhysRevD.83.046003

[37] M. Edalati and J.F. Pedraza, Phys. Rev. D 88, 086004 (2013), http://dx.doi.org/10.1103/PhysRevD.88.086004

[38] P. Dey and S. Roy, arXiv:1307.0195

[39]A. Karch, D.T. Son, and A.O. Starinets, Phys. Rev. Lett. 102, 051602 (2009), http://dx.doi.org/10.1103/ PhysRevLett.102.051602

[40] C.Hoyos, A. O'Bannon, and J.M.S. Wu, J.High Energ. Phys. 9, 086 (2010), http://dx.doi.org/10.1007 JHEP09(2010)086

[41]B. Swingle, L. Huijse, and S. Sachdev, arXiv:1308. 3234

[42]N. Ogawa, T. Takayanagi, and T. Ugajin, J. High Energ. Phys. 1, 125 (2012), http://dx.doi. org/10.1007/JHEP01(2012)125
[43] Y. Pomeau and P. Resibois, Phys. Rep. 19,63 (1975), http://dx.doi.org/10.1016/0370-1573(75)90019-8

[44] C. Hoyos, B.S. Kim, and Y. Oz, arXiv:1304.7481

[45] C. Hoyos, B.S. Kim, and Y. Oz, J. High Energ. Phys. 3, 029 (2014), http://dx.doi.org/10.1007/ JHEP03(2014)029

[46] C. Hoyos, B.S. Kim, and Y. Oz, arXiv:1312.6380

[47] M.H. Christensen et al., arXiv:1311.6471

[48] M.H. Christensen et al., Phys. Rev. D 89, 061901 (2014), http://dx.doi.org/10.1103/PhysRevD.89.061901

[49] A. Karch, arXiv:1405.2926

[50]P. Kovtun and A. Ritz, Phys. Rev. D78, 066009(2008), http://dx.doi.org/10.1103/PhysRevD.78.066009

[51] S. Jain, J. High Energ. Phys. 11, 092 (2010), http:// dx.doi.org/10.1007/JHEP11(2010)092

[52] R.A. Davison, K. Schalm, and J. Zaanen, arXiv: 1311.2451

[53]S.S. Pal, Phys. Rev. D 84, 126009 (2011), http:// dx.doi.org/10.1103/PhysRevD.84.126009

[54] K.-Y. Kim and D.-W. Pang, arXiv:1108.3791

[55]A. O'Bannon, Phys. Rev. D 76, 086007 (2007), http://dx.doi.org/10.1103/PhysRevD.76.086007

[56] N. Iqbal and H. Liu, Phys. Rev. D 79, 025023 (2009), http://dx.doi.org/10.1103/PhysRevD.79.025023

[57] K. Hashimoto and N. Iizuka, arXiv:1207.4643

[58] M.J. Luo, arXiv:1205.3267

[59]M.J. Luo, Mod. Phys. Lett. B 28, 1450005 (2014), http://dx.doi.org/10.1142/S0217984914500055

[60] K. Hashimoto, N. Iizuka, and T. Kimura, arXiv: 1304.3126

[61]D.N. Basov et al., Rev. Mod. Phys. 83, 471 (2011), http://dx.doi.org/10.1103/RevModPhys.83.471

[62] M. Mueller, J. Schmalian, and L. Fritz, Phys. Rev. Lett. 103, 025301 (2009), http://dx.doi.org/10.1103/ PhysRevLett.103.025301

[63] M. Mueller et al., Phys. Rev. B 78, 085416 (2008), http://dx.doi.org/10.1103/PhysRevB.78.085416

[64]M. Mueller et al., Phys. Rev. 78, 115406 (2008), http://dx.doi.org/10.1103/PhysRevB.78.115406

[65] M. Mueller et al., arXiv:0810.3657

[66] M. Mueller et al., arXiv:0910.5680

[67] V.P.J. Jacobs, S.J.G. Vandoren, and H.T.C. Stoof, arXiv: 1403.3608

[68] V.P.J. Jacobs, S. Grubinskas, and H.T.C. Stoof, arXiv: 1411.4051

[69]B. Goutéraux, arXiv:1401.5436

[70] M. Blake and A. Donos, arXiv:1406.1659

[71] H.K. Pal, V.I. Yudson, and D.L. Maslov, arXiv: 1108.2435

[72] H.K. Pal, V.I. Yudson, and D.L. Maslov, arXiv: 1204.3591

[73]D.L. Maslov, V.I. Yudson, and A.V. Chubukov, arXiv: 1012.0069

[74]B. Goutéraux, J. High Energ. Phys. 1, 080 (2014), http://dx.doi.org/10.1007/JHEP01(2014)080

[75] A. Lucas, S. Sachdev, and K. Schalm, arXiv:1401.7993

[76] A. Lucas and S. Sachdev, arXiv:1411.3331

[77] S.A. Hartnoll et al., arXiv:1401.7012 
[78]S.A. Hartnoll and D.M. Hofman, Phys. Rev. Lett. 108, 241601 (2012), http://dx.doi.org/10.1103/ PhysRevLett.108.241601

[79] K. Balasubramanian and C.P. Herzog, arXiv: 1312.4953

[80] S.A. Hartnoll and J.E. Santos, arXiv:1402.0872

[81] S.A. Hartnoll, arXiv:1405.3651

[82] A. Amoretti et al., arXiv:1411.6631

[83] R.A. Davison and B. Goutéraux, arXiv:1411.1062

[84]R.A. Davison, Phys. Rev. D 88, 086003 (2013), http://dx.doi.org/10.1103/PhysRevD.88.086003

[85]M.Blake andD.Tong, Phys. Rev.D 88, 106004(2013), http://dx.doi.org/10.1103/PhysRevD.88.106004

[86] T. Andrade and B. Withers, arXiv:1311.5157

[87]M. Blake, D. Tong, and D. Vegh, Phys. Rev. Lett. 112, 071602 (2014), http://dx.doi.org/10.1103/ PhysRevLett.112.071602

[88] A. Amoretti et al., arXiv:1407.0306

[89] M. Baggioli and O. Pujolas, arXiv:1411.1003

[90] A. Donos and S.A. Hartnoll, Nature Phys. 9, 649 (2013), http://dx.doi.org/10.1038/nphys2701

[91] P.M. Hogan andA.G. Green, Phys. Rev. B 78, 195104 (2008), http://dx.doi.org/10.1103/PhysRevB.78.195104

[92]J.-R. Sun, S.-Y. Wu, H.-Q. Zhang, Phys. Lett. B 729, 177 (2014), http://dx.doi.org/10.1016/j.physletb.2014.01.005

[93] M. Alishahiha, M.R.M. Mozaffar, and A. Mollabashi, arXiv:1208.2535

[94] M. Alishahiha, E. Colgin, H. Yavartanoo, J. High Energ. Phys. 11, 137 (2012), http:// dx.doi.org/10.1007/JHEP11(2012)137

[95]K.Goldstein etal., J.High Energ. Phys. 8,078 (2010), http://dx.doi.org/10.1007/JHEP08(2010)078

[96] S.S. Gubser and F.D. Rocha, Phys. Rev. Lett. 102, 061601 (2009), http://dx.doi.org/10.1103/ PhysRevLett.102.061601

[97] B. Goutéraux and E. Kiritsis, I. High Energ. Phys. 12, 036 (2011), http://dx.doi.org/10.1007 IHEP12(2011)036

[98] C. Charmousis et al., J. High Energ. Phys. 11, 151 (2010), http://dx.doi.org/10.1007/JHEP11(2010)151

[99] A. Donos and J.P. Gauntlett, arXiv:1401.5077

[100] B.-H. Lee, D.-W. Pang, and C. Park, arXiv:1006.1719

[101] B.-H. Lee, D.-W. Pang, and C. Park, arXiv:1006.0779

[102] B.-H. Lee, D.-W. Pang, and C. Park, Int. J. Mod. Phys. A 26, 2279 (2011), http://dx.doi.org/10.1142/ S0217751X11053481

[103]W. Witczak-Krempa and S. Sachdev, Phys. Rev. B 86, 235115 (2012), http://dx.doi.org/10.1103/ PhysRevB.86.235115
[104] W. Witczak-Krempa and S. Sachdev, Phys. Rev. B 87, 155149 (2013), http://dx.doi.org/10.1103/ PhysRevB.87.155149

[105] B.L. Altshuler, L.B. Ioffe, and A.J. Millis, Phys. Rev. B 50, 14048 (1994), http://dx.doi.org/10.1103/ PhysRevB.50.14048

[106]A.V. Chubukov, Physics 3, 70 (2010), http:// dx.doi.org/10.1103/Physics.3.70

[107]S.-S. Lee, Phys. Rev. B 80, 165102 (2009), http:// dx.doi.org/10.1103/PhysRevB.80.165102

[108] M.A. Metlitski and S. Sachdev, Phys. Rev. B 82, 075127 (2010), http://dx.doi.org/10.1103/ PhysRevB.82.075127

[109] M.A. Metlitski and S. Sachdev, Phys. Rev. B 82, 075128 (2010), http://dx.doi.org/10.1103/ PhysRevB.82.075128

[110] A.L. Fitzpatrick et al., arXiv:1307.0004

[111] A.L. Fitzpatrick et al., arXiv:1312.3321

[112]D.F. Mross et al., Phys. Rev. B 82, 045121 (2010), http://dx.doi.org/10.1103/PhysRevB.82.045121

[113]D. Dalidovich andS-S. Lee, Phys. Rev. B88, 245106 (2013), http://dx.doi.org/10.1103/PhysRevB.88.245106

[114]D.V. Khveshchenko, Phys. Rev. B 86, 115115 (2012), http://dx.doi.org/10.1103/PhysRevB.86.115115

[115] S.A. Hartnoll et al., Phys. Rev. B 84, 125115 (2011), http://dx.doi.org/10.1103/PhysRevB.84.125115

[116] A.V. Chubukov, D.L. Maslov, and V.I. Yudson, arXiv: 1401.1461

[117] M. Metlitski et al., arXiv:1403.3694

[118] C.M. Varma, Z. Nussinov, and W. Van Saarloos, Phys. Rep. 361, 267 (2002), http://dx.doi.org/10.1016/ 50370-1573(01)00060-6

[119] P. Gegenwart et al., Phys. Rev. Lett. 94, 076402 (2005),http://dx.doi.org/10.1103/PhysRevLett.94.076402

[120]A. Schoeder et al., Nature 407, 351 (2000), http://] dx.doi.org/10.1038/35030039

[121]].S. Helton et al., Phys. Rev. Lett. 104, 147201 (2010), http://dx.doi.org/10.1103/PhysRevLett.104.147201

[122]D. Pilon et al., Phys. Rev. Lett. 111, 127401 (2013), http://dx.doi.org/10.1103/PhysRevLett.111.127401

[123] M. Yamashita et al., Science 328, 1246 (2010), http://dx.doi.org/10.1126/science.1188200

[124]S. Elsasser et al., Phys. Rev. B 86, 155150 (2012), http://dx.doi.org/10.1103/PhysRevB.86.155150

[125]E. Abrahams, J. Schmalian, and P. Woelfle, arXiv: 1303.3926

\section{HOLOGRAFIJOS KRIZINĖS BŪSENOS TEORIJOS KRITIKA}

\section{D.V. Khveshchenko}

Šiaurès Karolinos universitetas, Chapel Hill, Šiaurés Karolina, Jungtinès Valstijos 